\title{
Mott Memory and Neuromorphic Devices
}

\section{Correlations of electrons - arising in structural and physical phase transitions - provide a nanoscale-compatible mechanism of possible utility to electronics. This paper discusses the mechanisms and their implications in memory and information processing.}

\author{
By You Zhou and Shriram Ramanathan
}

\begin{abstract}
Orbital occupancy control in correlated oxides allows the realization of new electronic phases and collective state switching under external stimuli. The resultant structural and electronic phase transitions provide an elegant way to encode, store, and process information. In this review, we examine the utilization of Mott metal-to-insulator transitions, for memory and neuromorphic devices. We emphasize the overarching electron-phonon coupling and electron-electron interaction-driven transition mechanisms and kinetics, which renders a general description of Mott memories from aspects such as nonvolatility, sensing scheme, read/write speed, and switching energy. Various memory and neuromorphic device architectures incorporating phase transition elements are reviewed, focusing on their operational principles. The role of Peierls distortions and crystal symmetry changes during phase change is discussed. Prospects for such orbitronic devices as hardware components for information technologies are summarized.
\end{abstract}

KEYWORDS | Complex oxide; emerging memory; metal oxide; metal-to-insulator transition; Mott insulator; neuromorphics; nonvolatile; orbitronics

\section{INTRODUCTION}

The scaling of semiconductor devices approaching 10-nm critical dimensions is much indebted to the success of the electronic band theory. Nevertheless, as early as 1937, de

Manuscript received September 28, 2014; revised April 1, 2015; accepted May 6, 2015 Date of publication June 26, 2015; date of current version July 15, 2015. This work was supported by the Army Research Office under Grant W911NF-14-1-0669; by the Air Force Office of Scientific Research under Grant FA9550-12-1-0189; and by the National Academy of Sciences.

The authors are with the John A. Paulson School of Engineering and Applied Sciences, Harvard University, Cambridge, MA 02138 USA (e-mail: youzhou@seas.harvard.edu; shriram@seas.harvard.edu).

Digital Object Identifier: 10.1109/JPROC.2015.2431914
Boer and Verwey noted that the band theory is insufficient to explain the electrical properties of many transition metal oxides with a partially filled $d$-electron band, which were expected to be metallic but are insulators in reality [1]. In 1949, Mott put forward a theory on how electronelectron interactions in these materials (commonly referred to as Mott insulators despite the fact that many of them display complex electronic phase diagrams that may not be wholly described by the original theory) could explain their insulating states [2]. It was later discovered that Mott insulators not only show unconventional electrical insulating behavior, but also often exhibit interesting phase transitions with drastic change in their electrical and magnetic properties under various stimuli. In the words of Nevill Mott, the Mott transition can strictly be defined as a "transition from an antiferromagnetic insulator to a metal" [3]. In this review, we use the terminology of Mott materials in a broader sense to describe materials with strong correlation that show insulator-to-metal transitions despite the fact that ordering of spins may not exactly coincide with electrical conductivity changes.

Recently, the potential applications of Mott insulators, especially those manifesting metal-to-insulator transitions, in memory devices have been actively explored, as the phase transitions could be triggered at subnanosecond timescales [4], [5] and the state of devices can be accessed electrically. It is also noteworthy that the electronic-structural phase changes in complex oxide thin films are robust and reversible for millions of cycles, in sharp contrast to bulk single crystals that shatter across the transition due to stress buildup. The enhanced reliability of phase transitions has contributed to the exponential growth of interest in this topic from not only applications' point of view but also the ability to study intrinsic physics of the transition with lowdimensional materials. In turn, this motivates state-of-theart experimental techniques such as ultrafast diffraction, spectroscopy and other in situ diagnostics to be applied to 
(a)

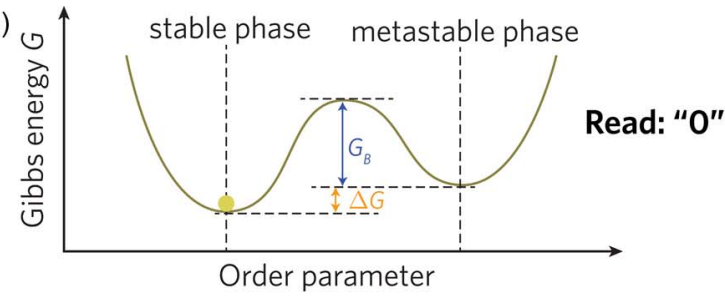

(b)

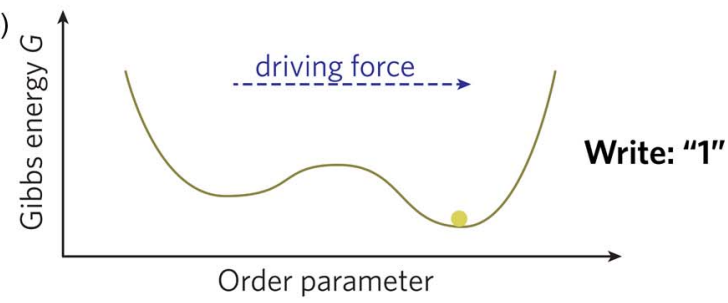

(c)

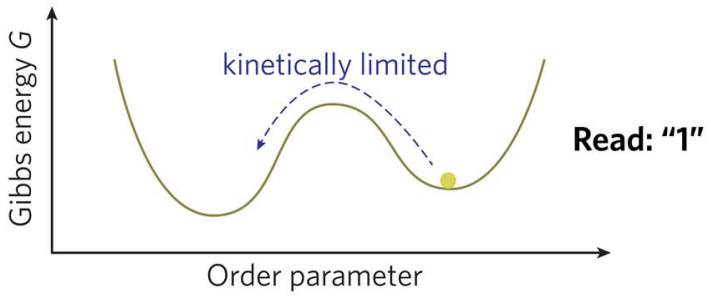

(d)

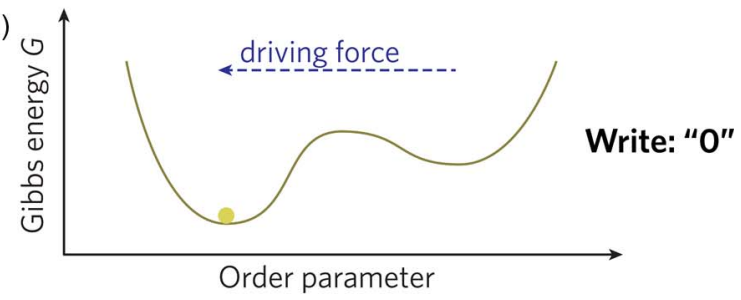

Fig. 1. Free energy landscape of Mott memories under writing and reading signals as a function of order parameter. (a) At the standby temperature, the system at equilibrium is in its thermodynamic stable phase (state " $O$ "). $\Delta G$ is the free energy difference between stable and metastable phases (state "1"). (b) An external stimulus drives the phase transition from state " 0 " to state " 1 " and the minimum energy cost and the minimum energy dissipated in the system is $\Delta G$. (c) When the writing signal is removed, the system in state " 1 " faces an energy barrier of $G_{B}$ and the relaxation to state "1" is limited by the kinetics of the transition. The relative magnitude of kinetic barrier $G_{B}$ and thermodynamic driving force $\Delta G$ determines whether the state is volatile. The memory is volatile if the metastable state " 1 " cannot be retained for a significant period of time. Otherwise, it can function as a nonvolatile memory and (d) another external signal is needed to change "1" back to " 0 ."

such problems, furthering our fundamental understanding of strong correlations in complex systems.

The general idea of utilizing Mott transition in memory devices is illustrated in Fig. 1 by considering the Gibbs free energy landscape of the system under write and read signals. Initially, the system is in the stable phase (state "0"). An external stimulus drives the phase transition from the thermodynamically stable phase to the metastable phase (state "1") (for example, from the insulating state to the metallic state). When the stimulus is removed, the relaxa- tion to state " 0 " is limited by the kinetics of the phase transition. If the kinetic barrier is much smaller than the thermodynamic driving force, the metastable state " 1 " could not be retained for significant period of time and the memory will be mono-stable and volatile. With a large kinetic barrier, on the other hand, the device would be quasi-bistable and nonvolatile, in which case another external stimuli is needed to switch from "1" to " 0 ." Theoretically, it is even possible to realize both volatile and nonvolatile operations with a single material at two different temperatures with different magnitude of thermodynamic driving force. By way of illustration, many of the phase transitions of Mott insulators are hysteretic at temperatures close to $T_{c}$, which could enable the nonvolatile operation. On the contrary, the stable phase will always be restored in the absence of external stimuli if the ambient temperature is outside the hysteretic window.

In comparison with memory devices like DRAM and SRAM, where the state is stored in the form of charge on capacitors, the state of Mott memories could be memorized and read out in the form of electronic and magnetic properties. This enables the compact packing of twoterminal cross-point array with $4 F^{2}$ cell area size ( $F$ is the minimum chip feature size). What is more, the speed of electrically triggered Mott transitions [6], [7] in twoterminal devices is comparable with standard memory devices including DRAM, SRAM, and faster than Flash [8], while the demonstrated speed of optically triggered switching can even approach the gate delay of the stateof-the-art transistors [9]. It has also been demonstrated that the write energy per transition is sub-100 $\mathrm{fJ}$ and could be scaled down with smaller device dimensions [7]. Additionally the write/read voltage values are compatible with typical supply voltages of silicon circuits.

The outline of the paper is as follows. Section II overviews the Mott transition phenomena and introduces the key mechanisms involving cooperative structural and electronic phase change to the general reader. Various devices that have been realized to date utilizing Mott transitions for memory and neuromorphic devices are discussed in Section III. Section IV evaluates and benchmarks the performance metrics of Mott transition memory devices, followed by a summary section.

\section{MATERIAL PROPERTIES}

\section{A. Metal-to-Insulator Transition Phenomena}

Several types of phase transitions involving change in magnetic, electric, and mechanical properties exist in the family of Mott insulators. The metal-to-insulator transitions refer to a class where the competition between the electron localization and delocalization lead to drastic changes in the conductivity of materials. These phase transitions can be triggered by thermal energy, electric fields, and optical stimuli, and the state of the system can be 


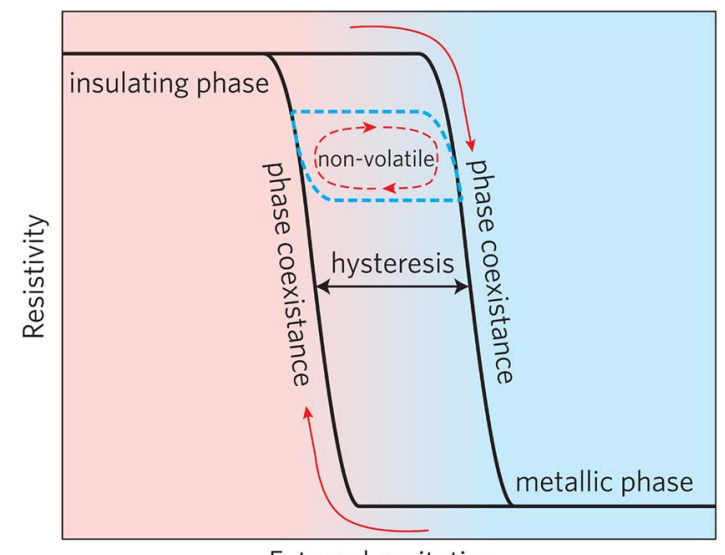

External excitation

(thermal, electrical, optical, mechanical and other)

Fig. 2. Generic features of metal-to-insulator transitions. The metalto-insulator transition in Mott insulators can be driven by various kinds of external perturbations. Above a threshold perturbation, the material resistivity changes drastically by several orders of magnitude. Near the threshold, phase coexistence of both insulating and metallic phase can occur. Many of the transitions also exhibit hysteresis. Within the hysteresis window, the system's resistance could be nonvolatile.

readily read out by electrical or magnetic signals. Fig. 2 illustrates some of the generic features of how the resistivity of the materials responds to external perturbations. 1) The system is initially in the insulating phase. As the external perturbation reaches a certain threshold value, a sharp phase transition is induced and the resistance drops by several orders of magnitude. When the external perturbation is removed, the system reverts back to the insulating phase. 2) The intermediate state between the insulator and metal is the coexistence of both phases [10]-[12], usually reflected by the sharp yet smooth change in resistivity as a function of perturbation. Transitions with no phase coexistence may be observed when the size of the device is smaller than the domain sizes. 3) Many transitions are hysteretic, meaning the perturbation strength to induce phase transition in one way is different from that in the other way. 4) Within the hysteresis window, the resistivity value depends on not only external field but also history and could be nonvolatile (dashed line and arrows in Fig. 2).

\section{B. Elementary Mott Transition Mechanisms}

In classical band theory, electrons could be treated as particles that move in an effective periodic potential independently from other electrons. Band forms from the overlapping atomic orbitals and the bandwidth $W$ is related to the system's energy gain from electron delocalization, or equivalently electron's kinetic energy that is often denoted as $t$. Materials with partially filled orbitals also have partially filled bands and are therefore metals. This criterion fails for Mott insulators, such as transition metal oxides with partially filled $3 d, 4 d$, or $4 f$ orbitals. In such materials, the Coulomb repulsion energy between electrons (electron correlation), often being referred to as $U$, is stronger than the kinetic energy $t$ and can therefore inhibit electrons from delocalizing to form bands or hopping through the lattice [Fig. 3(a)]. In the band picture, the original partially filled electronic band splits into one empty band and one filled band. The material is hence insulating.

The competition between carrier localization and delocalization drives the transition between insulating and metallic states. One way to induce such a phase transition is to tune the magnitude of delocalization energy $t$ or, in other words, the bandwidth W. For example, compressing the lattice constant by external stress can increase $W$ and leads to bandwidth-controlled MIT, as illustrated in Fig. 3(b). The other way is to change the effective $U$ between (a)

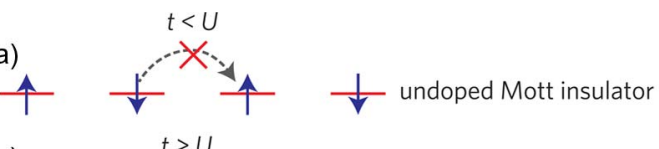

(b)

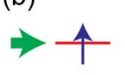

(c)

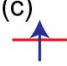

(d)
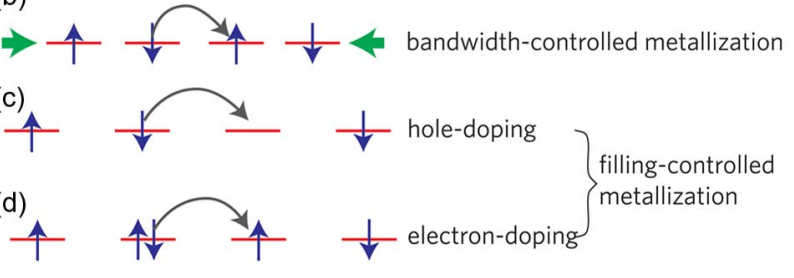

(e)

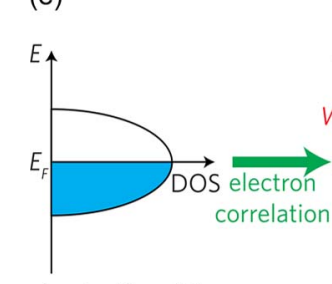

classical band theory
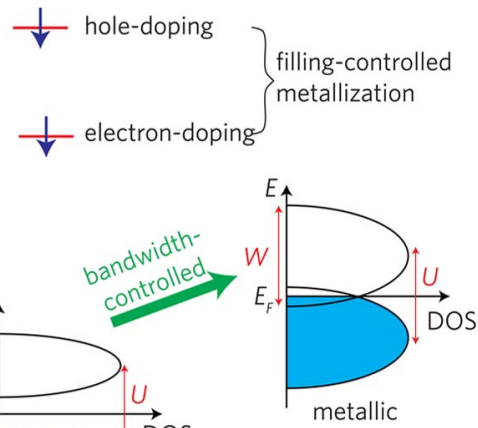

metallic

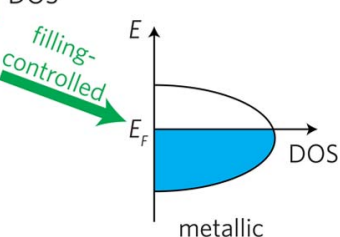

Fig. 3. Mechanisms of metal-to-insulator transitions in Mott insulators. (a) In the insulating phase, electron transport/hopping is forbidden because Coulomb repulsion between electrons $u$ is much larger than electron's kinetic energy $t$. (b) Bandwidth-controlled metal-to-insulator transition: decreasing the interatomic distance increases the electronic bandwidth and delocalization energy $t$ and drives an insulator-to-metal transition when $t$ becomes larger than $u$. (c) and (d) Filling controlled metal-to-insulator transition: hole- and electron-doping create empty or doubly occupied atomic sites, respectively. Since there is no more energy penalty for electron hopping to occur, the insulator becomes a metal. (e) The electronic band diagram evolution during metal-to-insulator transitions: classical band theory predicts that a Mott insulator has nonzero density of states at the Fermi level and is metallic. In reality, the band splits into two as a result of electron correlation and Fermi energy lies in the gap. Both increasing the bandwidth $w$ and introducing extra carriers can lead to the collapse of the bandgap. 
electrons. When electrons/holes are added into the system, the energy cost for hopping is reduced for certain electrons, reducing the effective Coulomb repulsion energy $U$ [Fig. 3(c) and (d)]. This can be also seen from the perspective that the added carriers can more effectively screen the electron-electron interaction and reduce $U$. The carrier-induced transition is referred to as fillingcontrolled MIT. In some cases, both mechanisms may come into play, e.g., certain temperature-driven transitions. The evolution of band structure across bandwidthcontrolled and filling-controlled MITs is shown in Fig. 3(e).

\section{Interplay Between Various Degrees of Freedom}

The so-called Mott transition is purely electronic and does not take other interactions into account. An important feature of Mott insulators in reality, however, is that the energy of many other interactions is on the same order of magnitude with electron-electron correlation $U$ and kinetic energy $t$, and therefore these interactions also become relevant in accurately dictating a material's electronic properties. These include electron-lattice, spin-spin, and other types of interactions. As a result, although intrinsic Mott transitions should be purely electronic in origin and not assisted by lattice, charge, and other degrees of freedom, the interplay between them leads to the observation that most of the electronic phase transitions in transition metal oxides are accompanied by structural and other types of transition. It is also important to note here that the structural symmetry changes might initiate electronic transitions or vice versa, so these can be inherently coupled. In this section, we consider examples where the interplay between different degrees of freedom is important in studying the MIT properties.

Since $d$ orbitals are degenerate [fivefold degenerate in spherical potential, and split into two levels in a tetrahedral or octahedral crystal field because of broken symmetry: $e_{g}$ level with twofold and $t_{2 g}$ level with threefold degeneracy, as shown in Fig. 4(a)], the localized $d$ electrons can occupy multiple possible orbitals, adding an orbital degree of freedom to the system. In addition, crystal structural change (the lattice degree of freedom) modifies the electronic band structure, which could be coupled with Mott transitions. For example, Fig. 4(b) shows how the elongation of metal-oxygen bonds along the $z$-axis of the $\mathrm{MO}_{6}$ octahedron, i.e., Jahn-Teller effect, can lift the degeneracy in $e_{g}$ and $t_{2 g}$ levels, which could lead to metal-to-insulator transitions for certain electron configurations [13]. In oxides with rutile structure, the interaction between metal ions in the edge-sharing $\mathrm{MO}_{6}$ octahedra also modifies the band structures, as shown in Fig. 4(c). The $d_{/ /}$orbital has electron lobes pointing along the rutile $c$-axis, whereas the $\pi^{*}$ orbitals have their electron lobes in the plane perpendicular to the $c$-axis. This unique aspect of band structure lays the foundation for the MIT in $\mathrm{VO}_{2}$, as will be discussed in detail in Section II-D.

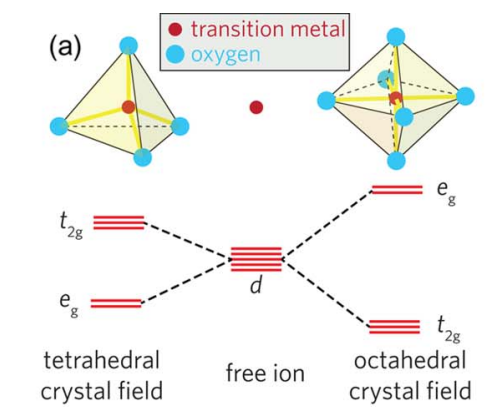

(b)
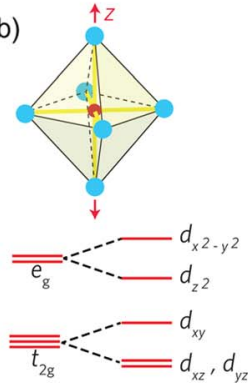

Jahn-Teller effect

(d)

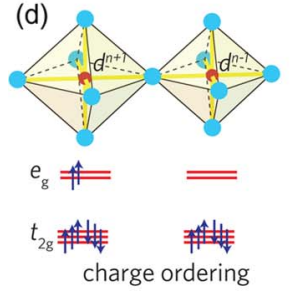

(c)
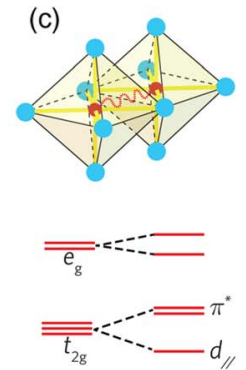

edge-sharing in rutile
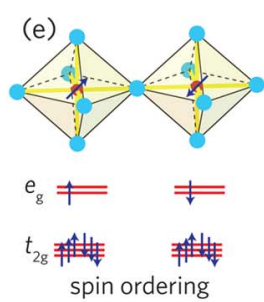

(f)

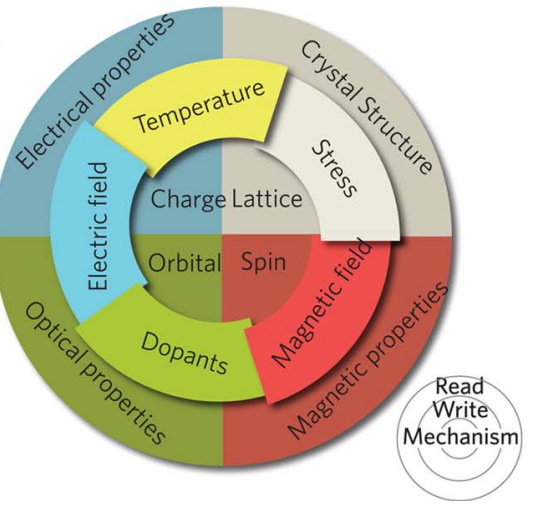

Fig. 4. Interplay between various degrees of freedom in Mott insulators. (a) Crystal field splits the originally fivefold degenerate $d$-orbitals into a twofold degenerate $e_{g}$ and a threefold degenerate $t_{2 g}$. The relative energy of $e_{g}$ and $t_{2 g}$ levels depends on the coordination number of the metal ion. (b) Jahn-Teller effect (elongation of $\mathrm{M-O}$ bond along the z-axis of the octahedron) and (c) metal-to-metal interaction in rutile (examples include $\mathrm{TiO}_{2}$ and the metallic state of $\mathrm{VO}_{2}$ and $\mathrm{NbO}_{2}$ ) can both further lift the degeneracy of $e_{g}$ and $t_{2 g}$ levels and modify the band structure. (d) Charge ordering and (e) spin ordering can also occur and influence the transport properties of correlated oxides. (f) In Mott insulators, different degrees of freedom are coupled together, making them sensitive to various perturbations and showing changes in different physical properties. The inner circle shows the coupling between various degrees of freedom. The mid-ring represents various ways to trigger phase transitions and the outer ring illustrates the physical properties that can be changed by the transitions. 
In addition to the lattice degree of freedom, long-range charge or spin ordering can also occur (charge and spin degrees of freedom). Fig. 4(d) and (e) gives examples of charge ordering and spin ordering in oxides with $d^{7}$ electron configurations. Charge ordering can lead to spatially varying metal-oxygen bond length and insulating electrical properties. A well-known example is the MIT in magnetite $\mathrm{Fe}_{3} \mathrm{O}_{4}$ discovered by Verwey [14]. It is also believed that charge-ordering plays an important role in the MIT of the rare earth nickelates [15]. Similarly, spin ordering also influences their electronic transport behavior, and is very often a natural result of charge localization. From the above discussion, it can be seen that the charge, lattice, spin, and orbital degrees of freedom are coupled in Mott insulators, which gives rise to complex phase diagrams [16]-[18]. For the same reason, the mechanisms of many phase transitions may be better explained by the cooperations between various interactions, rather than one single driving force [19].

The competition between different degrees of freedom enables one to trigger the transition by various means, as shown in Fig. 4(f), which allows flexibility in the operational principles of the Mott insulator devices. Similarly, the phase transitions can be reflected in change of different physical properties and therefore could be sensed with electrical, magnetic, or optical probes. For example, external magnetic fields can lead to large changes in the resistivity of some oxides, which is often called colossal magnetoresistance [20]. On the other hand, it is also possible to use an electric field to control the magnetization in the materials, also known as magnetoelectric effect [21], [22], which can be of interest in signal transduction or read out. Some Mott insulators exhibit ferroelectric properties, and can thus be integrated into the gate stack of conventional transistors for capacitance enhancement [23] or as a state register in memory. Because of the electron-phonon interaction, external stresses can also trigger changes in electronic/magnetic properties, which can be used as sensors for instance or mechanical energy harvesting or storage. Among the many methods to induce and sense the transitions, perhaps the most direct operation manner of a Mott-insulator-based memory device is to drive both the metal-to-insulator transition and sense the device conductance electrically. Yet the diversity of the MIT writing/ reading methods may add new functionalities to conventional memory technologies. The field of orbitronics explores such devices utilizing the orbital occupancy control in various materials.

\section{D. $\mathrm{VO}_{2}$ : A Case Study}

In this section, we examine a classical MIT material $\mathrm{VO}_{2}$, as an example to show how the lattice degree of freedom is coupled to the Mott transition. $\mathrm{VO}_{2}$ exhibits an insulator-to-metal transition accompanied by a structural transition with a transition temperature of $\sim 67^{\circ} \mathrm{C}$. The proximity to room temperature allows in-depth studies into the transition mechanism by a plethora of experimental techniques advanced in the past two decades. In contrast, $\mathrm{NbO}_{2}$ that undergoes insulator-to-metal transition at nearly $1083 \mathrm{~K}$ is far less studied and presents a formidable challenge. Simply retaining the phase and preventing oxidation to the more stable $5+$ valence of $\mathrm{Nb}$ at the thermal transition is a daunting problem. This compositional complexity will be discussed in Section II-G.

In its metallic phase, $\mathrm{VO}_{2}$ has a rutile structure (the socalled $\mathrm{R}$ phase), and the $d_{/ /}$and $\pi^{*}$ orbitals form two overlapping electronic bands, as shown in Fig. 5(a). If there are totally $N$ vanadium atoms, there are $N d$ electrons

(a) Metallic

Insulating
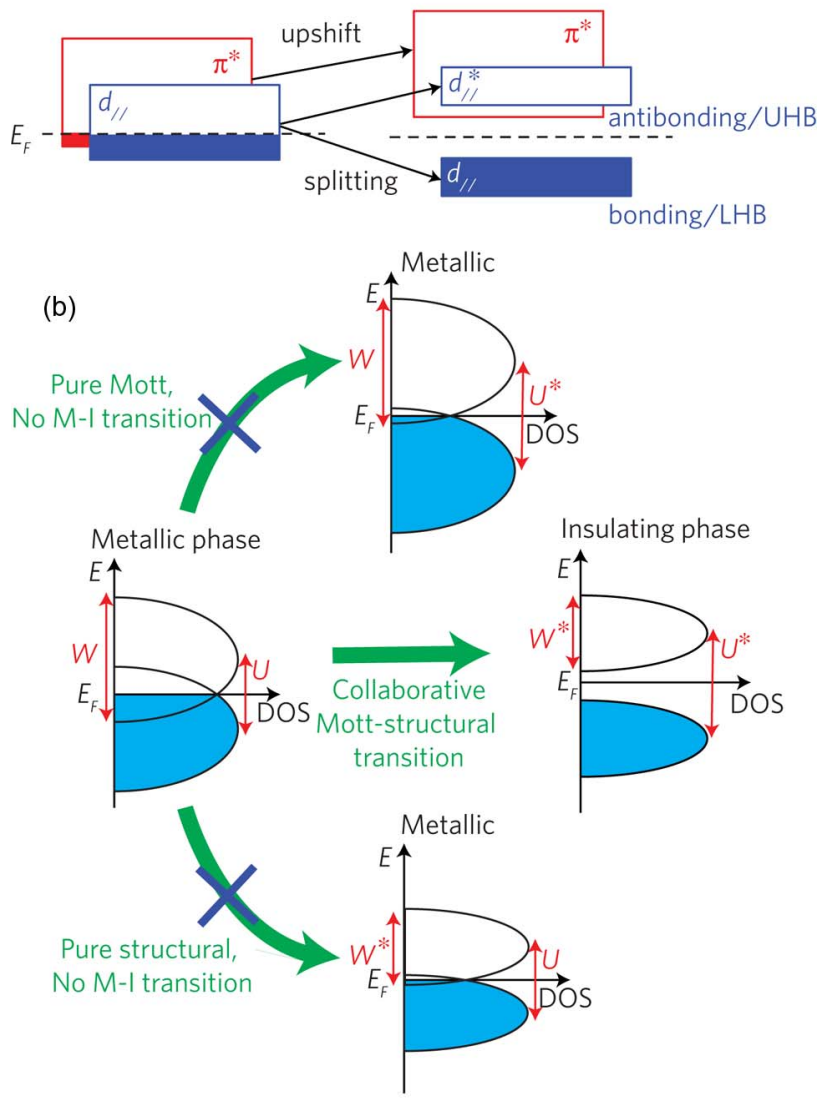

Fig. 5. Cooperative lattice and electronic phase change in correlated insulators. (a) Band structure of metallic $R$ phase and insulating M1 phase $\mathrm{VO}_{2}$. In the metallic phase, $d_{/ /}$and $\pi^{*}$ bands overlap with each other and both are partially filled. In the Goodenough picture (or Pierels picture), the dimerization of $\boldsymbol{V}$ atoms splits $\boldsymbol{d}_{/ /}$band into bonding and antibonding state and tilt of the dimers upshifts $\pi^{*}$ band. In the Mott picture, $\pi^{*}$ band is upshifted and depopulated from structural change, leading to less screened electron correlation and splits d// band into UHB and LHB. (b) Illustration of collaborative Mottstructural transition. A Mott transition reduces effective electron correlation $U$, but may not be enough to induce a metal-to-insulator transition. The decrease of bandwidth $W$ from structural change alone may also be insufficient to trigger the transition. $A$ simultaneous increase in $U$ and a decrease in $W$ can drive the transition in a collaborative fashion. 
and the total density of states in the overlapped band is $6 \mathrm{~N}$. The material is thus a metal. Across the electronic metalto-insulator transition, $\mathrm{VO}_{2}$ changes into a monoclinic structure characterized by vanadium dimerization and tilt of the dimers along the rutile $c$-axis (named M1 phase). In the Goodenough picture, the dimerization of $\mathrm{V}-\mathrm{V}$ pairs splits the $d_{/ /}$state into a bonding state and an antibonding state [24]. At the same time, the tilt of V dimers upshifts the $\pi^{*}$ bands. The combined structural change lifts the degeneracy and leads to a metal-to-insulator transition in Fig. 5(a). This picture is based solely on band theory and does not consider any electron correlation effect and often called Peierls transition. On the other hand, Zylbersztejn and Mott attributed the MIT mechanism to electron correlation [25]. They argued that the bandgap $(\sim 0.6 \mathrm{eV})$ cannot be quantitatively described by the Goodenough picture without taking into account the Hubbard U. In the Mott picture, the structural transition upshifts and depopulates the $\pi^{*}$ bands, making the electron repulsion on the $d_{/ /}$band less screened by the free carriers. The lower density of free electrons thus splits $d_{/ /}$band into the upper and lower Hubbard bands (UHB and LHB) and triggers the transition. Note that even in Mott's original picture, the structural transition is an important factor in explaining the transition [25]. For the past few decades, the nature of the MIT in $\mathrm{VO}_{2}$ has been heavily discussed [25]-[30]. Later observations of another monoclinic insulating phase (M2 phase) under doping and stress have further complicated the interpretation [31], [32]. Many recent studies point to a combined effect of lattice distortion and Coulomb correlation [30], [33]-[39], since neither Mott nor Peierls mechanism could alone explain the wide range of phenomena in $\mathrm{VO}_{2}$.

The phase transition dynamics studies reveal important knowledge on the following questions: Does the electronic transition happen before the structural transition? It has been found that the transition from initial insulating to the stable metallic state can be mediated through a metastable metallic phase [40]. This intermediate phase is structurally different from both the normal metallic and insulating phases. It is characterized by local atomic motion and the dilation of the vanadium-vanadium bond. The intermediate phase finally transits into the stable rutile phase. This shows that the electronic transition can happen before the full structural transitions, yet still coupled partly to it. To what degree can the electronic and structural transition be decoupled? Is it possible to achieve and also reverse the MIT without structural changes in $\mathrm{VO}_{2}$ ? There are several studies suggesting that there is a monoclinic metallic phase [41]-[48], which points to the possibilities of pure electronic transitions. The transitions could have different trajectories in phase space depending upon the experimental conditions and driving force. Some of the observations were made under nonequilibrium conditions [49] or with multiple driving forces, such as in thermally triggered MIT of strained $\mathrm{VO}_{2}$ [42], [46], [47]. The nature of the mono- clinic metallic phase, however, also needs to be carefully studied. Most X-ray diffraction studies only monitor the out-of-plane lattice constant change, and therefore do not provide full information on the whole crystal structure. Future works studying the entire reciprocal space could help to resolve the issue of whether there is absolutely no structural change and an intrinsic pure Mott transition can be induced in $\mathrm{VO}_{2}$ by various driving forces. In addition, the interpretation of experimental results is complicated by phase coexistence [10], [50], a generic feature of first-order phase transitions, near the transition temperature. Using $\mathrm{X}$-ray diffraction mapping, it has been shown that only lowtemperature monoclinic and high-temperature tetragonal phases exist at the transition temperature, and there is no evidence for the presence of $V$ dimers above the transition temperature in the thermally driven transition [51].

Fig. 5(b) illustrates the generic idea of a Mott transition coupled with a structural transition. In the metallic phase, the electron correlation energy corresponds to an effective Coulomb repulsion $U$ that is screened by the free electrons. The bandwidth $W$ is determined by the structure. In a pure Mott transition, the electron correlation energy increases, for example, by freezing out the free carriers by cooling. If bandwidth $W$ is, however, still larger than the unscreened Hubbard $U^{*}$, the MIT cannot be induced solely by electron correlation. On the other hand, a structural change can reduce the bandwidth to a lower value $W^{*}$, but may also not induce an MIT unless $U$ is small. By combining both effects, i.e., reduction of bandwidth and increase in the correlation, it then becomes possible to open up a bandgap and create an insulating phase. Of course, this picture does not provide a universal description of all the MIT phenomena. As discussed, a Mott transition in reality could be accompanied by symmetry breaking or change in the other degrees of freedom as well, and the structural change does not only modify bandwidth $W$, but also the band's relative position to the Fermi level. Therefore, each material may require specific detailed investigations. Suppose that if an external driving force such as strain is used to mimic the role of structural change (e.g., to modify the bandwidth), it then becomes possible to induce a pure Mott transition without the help of structural transition. This presents a promising route to explore purely electronic transitions, and the use of low-dimensional materials can contribute to advancing this frontier.

\section{E. Surface Versus Bulk Effects}

Surfaces of transition metal oxides can display properties distinct from bulk [52]. First, because of the net charges on the cation and oxygen anion, the surface of the crystal can exhibit a dipole, which is referred to as a polar surface. Polar surfaces are in general less stable than nonpolar surfaces and usually go through reconstruction to compensate the dipole moment. Second, the degree of coordination is unsaturated for the surface ions. Dangling bonds could interact with molecules in the environment if 
the surface is exposed. The unsaturation and dangling bonds will change the orbital thermodynamics in Fig. 4(a) and could lead to different electronic structure at the surface from the bulk. Third, as defects such as oxygen vacancies are more likely to form on the surface, they will also change the surface of the electronic structure. The scaling of the metal-to-insulator transitions discussed in this review can be imposed by the feasibility of retaining the same crystal structure in lower dimensions as the bulk. When scaling down the material, the total Gibbs free energy will be dominated by the surface rather than the bulk energy. As a result, the thermodynamically stable phase for lowdimensional oxides can be different from the bulk. All of the above surface effects could be coupled to other degrees of freedom, leading to emergent surface properties, although the fundamental physics is unchanged.

For $\mathrm{VO}_{2}$, it has been noted that both the insulating and metallic phases as well as the transition mechanism remains the same as the bulk for thin films down to $\sim 4 \mathrm{~nm}$, which suggests $\mathrm{VO}_{2}$ can be scaled down to such sizes without losing the functionality arising from phase change [53]. In $\mathrm{VO}_{2}$ nanowires, it has been observed that the $\mathrm{VO}_{2}$ surface stress can stabilize M2 phase [54], although the question whether there will be fundamentally different phase at the surface from the bulk remains to be explored. Another example is the observation of a pure Mott transition without structural change at $\mathrm{Ca}_{2-\mathrm{x}} \mathrm{Sr}_{\mathrm{x}} \mathrm{RuO}_{4}$ surface [55]. In the low $\mathrm{Sr}$ doping regime, bulk $\mathrm{Ca}_{2-\mathrm{x}} \mathrm{Sr}_{\mathrm{x}} \mathrm{RuO}_{4}$ exhibits a metal-to-insulator transition and a concomitant structural change at $\sim 154 \mathrm{~K}$ with a Mott insulating ground state. It has been demonstrated that the structural distortion is important to create and stabilize the Mott insulating state in the bulk [56]-[58]. By studying the electrical properties and crystal structures of $\mathrm{Ca}_{2-\mathrm{x}} \mathrm{Sr}_{\mathrm{x}} \mathrm{RuO}_{4}(\mathrm{x}=0.1)$ surface, it was shown that the surface of the material goes through MIT at lower temperature $(\sim 130 \mathrm{~K})$ and there is no related structure change [55]. It is found that the surface $\mathrm{RuO}_{6}$ octahedra have larger rotational distortion and tilt than the bulk parts, which helps to narrow the $t_{2 g}$ bandwidth. Presumably these effects increase the ratio of Coulomb $U$ to bandwidth $W$, enabling the Mott transition without structural change.

On the other extreme, growing thick films of phase change materials approaching a micrometer or more remains a challenge due to the slow growth rates and retaining the single phase or stoichiometry. Self-annealing during the long growth cycles can lead to through-thickness compositional changes. Such thick films are gaining importance in the field of nonlinear optical materials and optical limiters and will require much effort in synthesis.

\section{F. Electrically Triggered Metal-to-Insulator Transition}

Fig. 6(a) shows typical current-voltage curves of E-MITs in two-terminal Mott metal-oxide-metal devices (e.g., $\mathrm{VO}_{2}$ [59], $\mathrm{V}_{2} \mathrm{O}_{3}$ [60], $\mathrm{NbO}_{2}$ [7], manganites [61], (a)

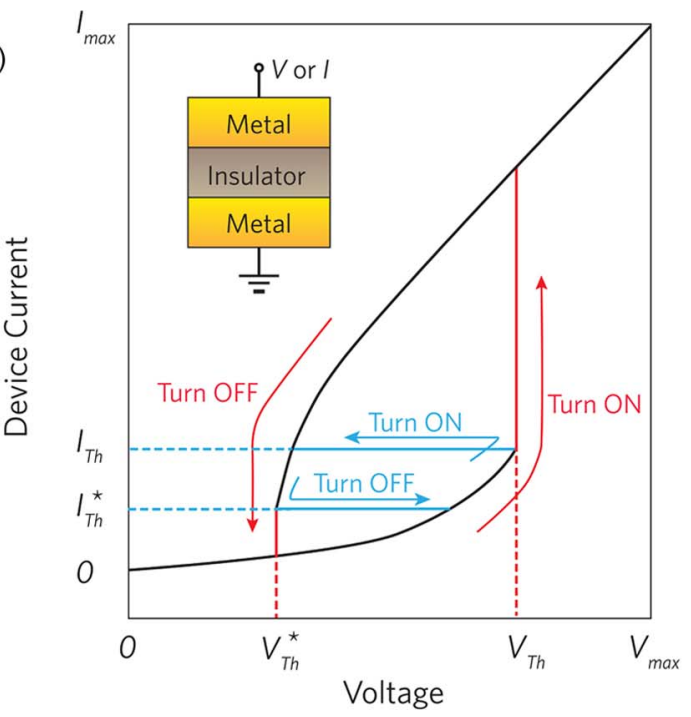

(b) hot carrier emission

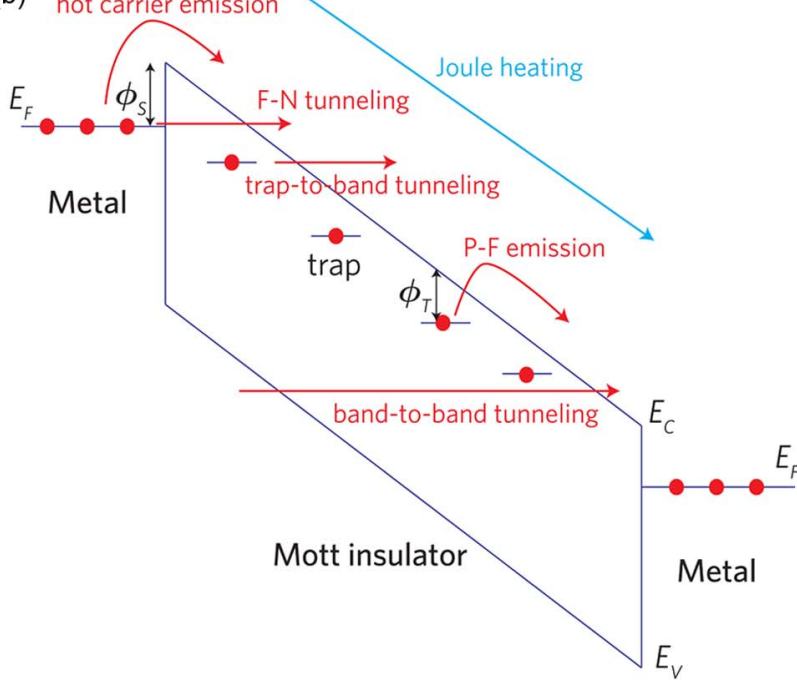

Fig. 6. (a) Typical current-voltage curves of electrically triggered metal-to-insulator transitions either driven by voltage or current source. (b) Possible mechanisms of the electrically triggered transition include: thermal Joule heating, and carrier injection due to Schottky thermionic emission, Fowler-Nordheim tunneling, Poole-Frenkel emission, trap-to-band tunneling, and band-to-band tunneling. Some of the processes require formation of Schottky barriers or presence of trap states.

magnetites [62], and $\mathrm{La}_{2-\mathrm{x}} \mathrm{Sr}_{\mathrm{x}} \mathrm{NiO}_{4}$ [63]). In the lowvoltage region, the device shows linear $I-V$ curve as in its insulating phase. Under large bias, the $I-V$ curve begins to deviate from linearity and above a certain threshold voltage $V_{\text {th }}$, the device resistance drops drastically. The corresponding threshold electric field is typically $10^{4}-10^{5} \mathrm{~V} / \mathrm{cm}$ [6], [7], [59], [60], [64]. The $I-V$ curve becomes linear again well above $V_{\mathrm{th}}$, and the device is switched on. When voltage is ramped down, the device turns ofF at $V_{\mathrm{th}}^{*}$ that is smaller than $V_{\text {th }}$, showing a hysteresis in the $I-V$ curve. Such switching behavior is often referred to as "threshold 
switching." In addition to voltage triggering, E-MIT can be also driven by a current source, which exhibits currentcontrolled negative differential resistance (CC-NDR) phenomena near the threshold current $I_{\mathrm{th}}$, as shown in Fig. 6(a) [65], [66]. In a symmetric device, the $I-V$ curve is independent of the voltage polarity. The threshold switching is due to metal-to-insulator transition and not defectrelated conductive filament formation as in RRAM based on the following observations: 1 ) the ON/OFF ratio of E-MIT matches the transition magnitude of thermal MIT [67]; and 2) the on/OFF resistance scales with the device area [6]. Both suggest bulk MIT instead of defect-related conductive filament (CF) formations [68]. Note that sometimes there will be multiple jumps in the $I-V$ curves, due to partial switching in multiple domains [67], [69]-[71]. The cross section of such conductive path is usually on the order of micrometers to millimeters and comparable with the device area [70], [71], in sharp contrast to the nanoscale CFs in RRAMs. Additionally, two terminal Mott switches could also be multileveled when taking advantage of the hysteresis of the transitions [72]. In samples synthesized with structural and compositional disorder, it is indeed possible to form local switching mediated by point defects that show bipolar switching behavior [73], [74]. The E-MIT is also different from that of phase change random access memories (PCRAMs). In PCRAMs, resistive switching is achieved by switching between amorphous and polycrystalline phases such as in chalcogenides [75], [76].

Several mechanisms could be responsible for inducing E-MITs, as illustrated in Fig. 6(b). First, Joule heating as a result of the current passed through can elevate the device temperature and drives MIT. Additionally, since the change in the carrier density can also cause a Mott transition by filling control, any process that introduces extra carriers in the conduction/valence band may also induce EMIT. Such effects include: 1) Fowler-Nordheim tunneling through or thermionic emission across a Schottky barrier if the metal/insulator contact is non-Ohmic [77]; 2) PooleFrenkel emission or trap-to-band tunneling in presence of defect states [78]-[80]; and 3) band-to-band tunneling, etc. It has been argued that Joule heating could not alone cause the transition [81]-[83], and the nonlinearity of $I-V$ curves just below $V_{\text {th }}$ could also be a signature of Poole-Frenkel emission [79], [80]. Other studies, based on the measurement of the device local temperature [84] or thermal modeling, especially considering the effect of phase coexistence [70], [71], [85], [86], point out contribution from thermal effects in direct current (dc) E-MIT [85]-[88]. Furthermore, the temperature rise as a result of Joule heating may also result in nonlinear $I-V$ curves. In a dc measurement, even if E-MIT were initially triggered electronically, the large on-state current would induce large local temperature change. Therefore, one needs to go to high fields and short pulses to minimize the heating energy dissipated in order to study the E-MIT mechanisms, as will be discussed in Section IV-A.
Other types of switching behavior can also be observed in two-terminal devices. Nonvolatile switching has been observed in a family of Mott insulators $\mathrm{AM}_{4} \mathrm{X}_{8}(\mathrm{~A}=\mathrm{Ga}$, $\mathrm{Ge} ; \mathrm{M}=\mathrm{V}, \mathrm{Nb}, \mathrm{Ta} ; \mathrm{X}=\mathrm{S}, \mathrm{Se}$ ), which was attributed to electrically triggered MIT [89]-[91]. Further studies are needed to address the questions on what is the relative importance of the electron correlation versus direct structural change as in PCRAMs. Multistate nonvolatile resistance change has been achieved in $\mathrm{VO}_{2}$ through Joule heating in the hysteresis window [72].

In a three-terminal transistor-type devices, there is yet another way to induce carrier density change, i.e., electrostatic carrier doping through field effect, which would not involve Joule heating because of low gate-drain current [92]. Attempts to trigger Mott transitions in solid-state field-effect transistors have been made with materials like $\mathrm{VO}_{2}$ [81], [93], [94], cuprates [95], and other correlated oxides [92]. However, the areal carrier density induced with solid dielectrics is usually insufficient to drive the phase transitions in most cases [96], [97]. Recently, there has been growing interest in using liquid-based electrolyte gating in electric double-layer transistors (EDLTs) to create high-density surface charge and, therefore, phase transitions [98]-[101]. Many such devices show hysteretic gating behaviors [102]-[104] that are of relevance to nonvolatile memory. The origin of hysteresis in such devices, however, is related to the compositional changes occurring at the electrolyte-oxide interface and distinct from undercooling mechanisms [105], [106].

\section{G. Materials Synthesis}

A problem often encountered in the growth and fabrication process of Mott insulators is that the MIT transition oN/OFF ratio is often smaller in thin films than in single crystals or becomes degraded after fabrication. This originates from the fact that transition metals can usually have multiple valence states, and there could be multiple crystallographic phases for a single stoichiometry. The anisotropy of crystal phases also leads to differences in ease of ion migration and therefore different activation barriers for oxidation/reduction of the anion sublattice. The interplay between different degrees of freedom makes the electrical properties extremely sensitive to deviation from stable crystal structures, nonstoichiometry, and defects. The ability to modify crystal composition by interstitial doping or metastable incorporation of dopants can lead to emergence of new electronic phases. Consequently, the growth of these materials often requires careful control of temperature, stoichiometry, and defects to achieve large on/OFF ratio. Significant progress has been made on both epitaxial [107]-[109] and nonepitaxial growths [110] of high-quality Mott insulators by PLD [111]-[113], sputtering, e-beam evaporation [114], atomic layer deposition [115], [116], chemical vapor deposition [117], [118], molecular beam epitaxy [119], [120], and sol-gel methods [121] in the form of thin films [122]-[125] and 
nanostructures [126]-[130]. Low-temperature deposition techniques combined with postannealing procedures have also been widely studied [115], [131]. Recently, it was demonstrated that the resistivity of $\mathrm{SmNiO}_{3}$ can change by eight orders of magnitude by electron doping, many orders of magnitude larger than its thermal MIT [132]. Such colossal resistance change is due to the band structure change of Mott insulators upon carrier doping. This can inspire the idea of using reversible ionic doping (controlled by electric field and independent of temperature) to achieve larger ON/OFF ratio than what is typically achievable in a thermal phase transition and forms the basis for a new research direction.

\section{MOTT-INSULATOR-BASED MEMORY DEVICES}

\section{A. Mott Selectors in 1S1R Arrays}

Mott insulators may be utilized as selector devices in the cross-point nonvolatile memory arrays such as for RRAM or PCRAM. Cross-point arrays shown in Fig. 7(a) offer one of the densest packing for nonvolatile RRAM and PCRAM with a cell size as small as $4 F^{2}$. However, with a single RRAM or PCRAM as the memory unit, the current can sneak through undesired pathways, leading to read/ write errors. As a result, a selector device with either nonlinear or asymmetric current-voltage characteristics (e.g., a diode or a transistor) needs to be integrated with each RAM cell to form a 1-selector-1-RRAM (1S1R) array [133]. Fig. 7(b) shows a 1S1R cross-point array with Mottinsulator-based threshold switches as the selectors. Because there are no junctions in Mott-insulator-based selectors, such structures could be more stackable, more compact, and easier to fabricate than typical diodes and transistors. What is more, Mott selectors may be especially useful for bipolar switching RRAMs as they allow large current to pass in both directions unlike diodes. As a result, there have been several studies investigating selector properties of $\mathrm{VO}_{2}$ and $\mathrm{NbO}_{2}$ [134]-[144].

The operational principle of a selector is as follows.

1) Reading of an HRS unit: During this step, when a read voltage $V_{\text {read }}$ is applied to the selected HRS cell, the total current needs to be low. A large sneak current will appear if all of the other cells are oN. In such an extreme case, the maximum voltage drop on any unselected cell will be half of the read voltage in a large array. Now if a Mott selector is added to each of the memory cell and its threshold voltage $V_{\text {th }}>1 / 2 V_{\text {read }}$, all the unselected Mott selectors stay off. In this way, the sneak current will be limited by the off resistance of the Mott selectors. Note that the Mott selector of the chosen HRS can still be turned on without setting the memory to LRS if $I_{\text {th }}$ is lower than $I_{\text {set }}$, and it is usually the case. In addition, the Mott selector of the chosen HRS could, in principle, (a)

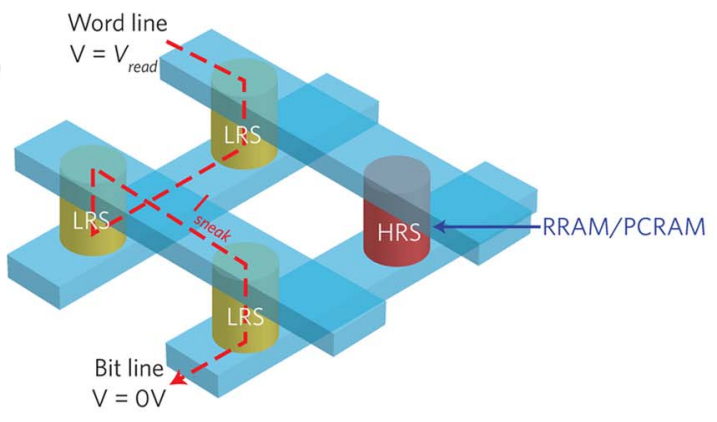

(b)
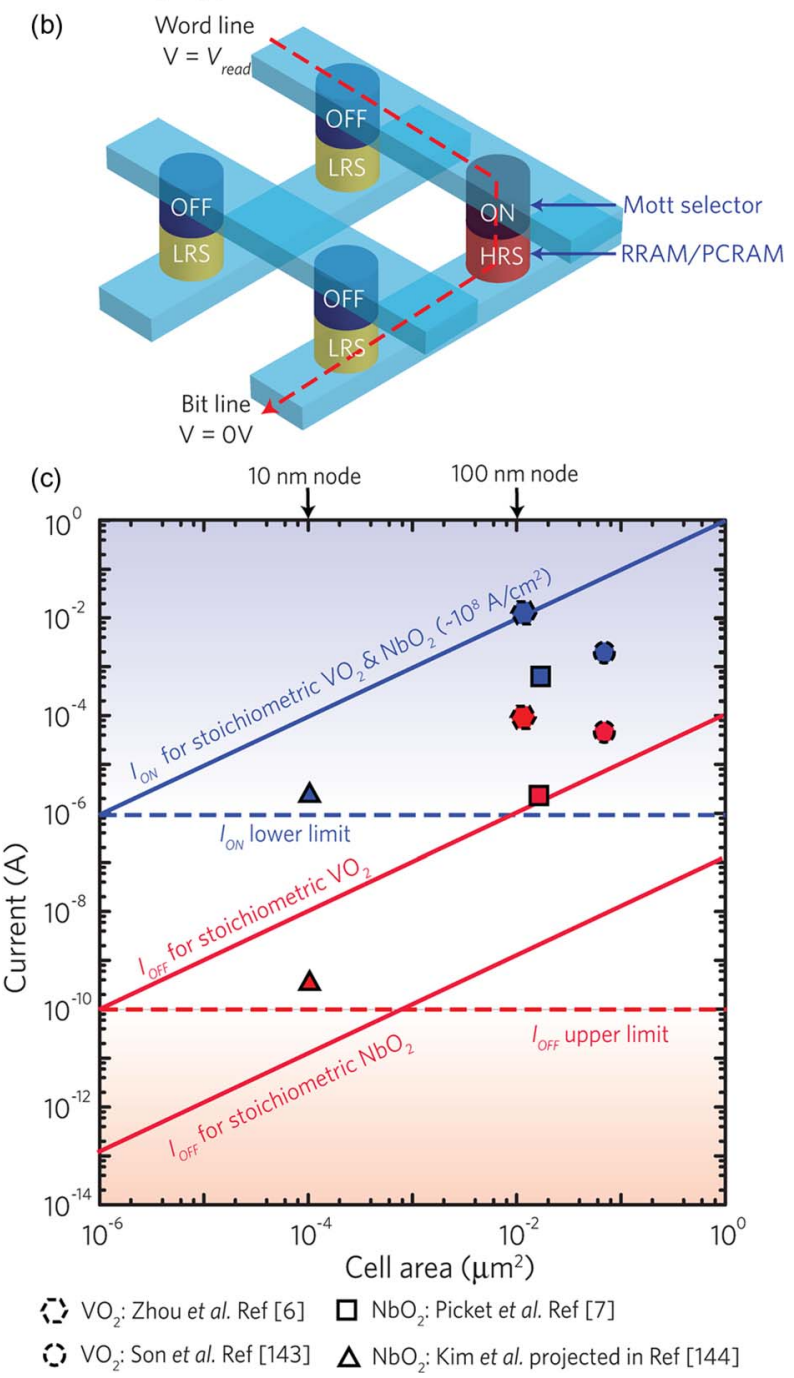

Fig. 7. (a) Cross-point array enables high-density stackable packing of nonvolatile memories (RRAMs or PCRAMs), but suffer from sneak path current problems when each unit only contains a memory. (b) Using Mott two-terminal threshold switches as selectors can prevent sneak current problems. Note that the selected Mott switch does not necessarily have to be ov if the selected memory is in the HRS state. (c) The design space of Mott selector devices considering the criterion for the on/off current. The weak scaling or nonscaling of on resistance and current due to the filamentary conduction in RRAMs imposes strict restrictions on the material choice for Mott selector devices whose conductance scales with device area. Experimental data are taken from [6], [7], [143], and [144]. Blue filling corresponds to on current while the red symbols represent off current. 
stay OFF, as the total current is distinguishable from the on-state.

2) Reading of an LRS unit is trivial. When the selected RRAM is in its LRS state, the selector will not impede the current flow as long as $V_{\text {th }}<V_{\text {read }}$.

3) Setting/resetting (writing) the unit: in the writing process, the current through the memory and selector should be large enough not only to turn the selector ON, but also to SET/RESET the memory. This requires the selectors to have relatively large current density in the ON-state, because of the relative large SET/RESET current of the RRAM. There are ongoing efforts in reducing the SET/ RESET current, which will not only put less restrictions on the selector and related circuit design, but also lowers the power consumption [145]. Another restriction is that the write voltage however should not be too large to change the state of nonselected memories in the sneak paths. A simple implement would require $V_{\text {write }}<2 V_{\text {th }}$, while less strict criterion could also be designed depending on the resistance ratio of the memory and selector elements.

Mott insulator selectors and RRAM have significantly different scaling behaviors (see Table 1) because of their different resistive switching mechanism. Since the threshold switching in Mott insulators is due to the bulk phase transition between the insulating and metallic phase, both the ON-state and OFF-state resistances will scale with device area as for a simple rectangular resistor with uniform resistivity. On the contrary, resistive switching in RRAM is facilitated by the formation and rupture of conductive filaments, leading to the weak scaling or even nonscaling of the SET/RESET current and ON-state resistance, etc. (see [145], [146], and reference therein). In principle, the CF conduction in RRAM would also confine the current flow cross section in the selector. In the following discussion, we assume no such confinement effect, which can be achieved by, e.g., inserting a metal electrode between the memory and the selector.

Of course, besides the threshold voltage, resistances of the RRAM and selector have to match with each other in the 1R1S memory arrays. The resistance of the HRS and

Table 1 Summary of Scaling Behavior of RRAM and Mott Selector

\begin{tabular}{|ccc|}
\hline \hline & & \\
& RRAM [145] & Mott selector \\
\hline Lateral dimension & $\lambda$ & $\lambda$ \\
Vertical dimension & $\lambda$ & $\lambda$ \\
High resistance & $1 / \lambda$ & $1 / \lambda$ \\
Low resistance & 1 & $1 / \lambda$ \\
$V_{T h}$ & $\mathrm{~N} / \mathrm{A}$ & $\lambda$ \\
RESET current & Weak scaling & $\mathrm{N} / \mathrm{A}$ \\
ON current & N/A & $1 / \lambda^{2}$ \\
\hline \hline
\end{tabular}

the LRS of the memory unit is referred to as $R_{\text {HRS }}$ and $R_{\mathrm{LRS}}$, respectively, while the resistance of the on-state and the OFF-state of the Mott selector is denoted as $R_{\mathrm{ON}}$ and $R_{\mathrm{OFF}}$. Fig. 7(c) illustrates the design space of Mott selectors in RRAM 1S1R arrays. First, the oN-state current of a selector has to be high enough to SET/RESET the memory unit. The program (SET/RESET) current of RRAM is typically $\sim 1-100 \mu \mathrm{A}$ independent of the cell size [145], [147]-[153], which sets the 1- $\mu$ A lower limit of the oNstate current of the Mott selector, as shown in Fig. 7(c). On the other hand, the OFF-state current of the selector must be low enough to prevent error readings due to the sneak current, especially when there are a large number of memory units in the 1S1R array. The total read current $I_{\text {read_H }}$ is the current across the selected HRS unit plus the sneak current paths across all other memory units $\left(I_{\text {read_H }}=I_{\text {HRS }}+I_{\text {sneak }}\right)$. The current across the selected HRS unit is given by $I_{\mathrm{HRS}}=V_{\text {read }} / R_{\mathrm{HRS}}$, because the selector is switched on by $V_{\text {read. }}$. In a 1 S1R array with $n \times n$ units ( $n$ being 1024 typically), the sneak current can be calculated from $I_{\text {sneak }}=V_{\text {read }} / R^{\prime}$, where $R^{\prime}$ is the effective resistance of the nonselected resistor network. The effective resistance of the remaining network will be the largest if all other memory units are in LRS except the selected one and can be estimated to be $R^{\prime}\left(R_{\mathrm{LRS}}+R_{\mathrm{OFF}}\right) / n$.

In order to be able to preserve the same ON/OFF ratio of the RRAM or PCRAM in a circuit as in single device, the sneak current has to be smaller than the HRS current, $I_{\text {sneak }}<I_{\text {HRS }}$. In other words, the sneak current across a single nonselected cell must be $n$ times smaller than the read current of the selected HRS cell in an $n \times n$. If $R_{\mathrm{HRS}}>10^{3} R_{\mathrm{LRS}}$, this leads to $R_{\mathrm{OFF}}>10^{6} R_{\mathrm{LRS}}$. However, we could, in fact, impose a less strict requirement on the sneak current. As long as the total current HRS read current $\left(I_{\text {read } \_H}=I_{\text {HRS }}+I_{\text {sneak }}\right)$ is ten times smaller than the LRS read current, i.e., $I_{\text {read } \_}>10 I_{\text {read } H} \sim 10 I_{\text {sneak }}$, it would be sufficient to differentiate the resistance states of the memory. This means that the insulating resistance of the selector must satisfy $R_{\mathrm{OFF}}>10^{4} R_{\mathrm{LRS}}$. The above analysis sets the upper limit of the OFF-state current of about $0.1 \mathrm{nA}$, as illustrated in Fig. 7(c).

As a result, a selector cell must have an on current over the horizontal dashed blue line and an off current below the dashed red line. For a Mott selector, the on/ofF-state current is given by $I=j A=\sigma E A$, where $A$ is the cell size, $j$ is the current density, $E$ is the applied electric field, and $\sigma$ is the conductivity in the corresponding phase. The read electric field $E$ should be comparable to the switching threshold $E_{T}$ (typically $10^{4}-10^{5} \mathrm{~V} / \mathrm{cm}$ ) and be constant as the driving voltage scales with the vertical dimension. The resistivity of single crystal $\mathrm{VO}_{2}$ for example changes from $10 \Omega \cdot \mathrm{cm}$ at room temperature to $\sim 10^{-3} \Omega \cdot \mathrm{cm}$ above the transition temperature in the metallic phase. Plugging in the values of resistivity of both phases and the electric field, we can plot both $I_{\mathrm{ON}}$ and $I_{\mathrm{OFF}}$ versus cell size curves for a $\mathrm{VO}_{2}$ selector device, as shown in Fig. 7(c). Because 
the resistivity of nonstoichiometric $\mathrm{VO}_{2}$ is lower than that of stoichiometric $\mathrm{VO}_{2}$ in the insulating phase and higher in the metallic phase, $I_{\mathrm{ON}}$ and $I_{\mathrm{OFF}}$ versus cell size curves practically set the upper limit of $I_{\mathrm{ON}}$ and the lower limit of $I_{\mathrm{OFF}}$ of a $\mathrm{VO}_{2}$ selector. The region between these two lines is the achievable design space for $\mathrm{VO}_{2}$ selectors. From Fig. 6(c), it is clear that $I_{\mathrm{ON}}$ of $\mathrm{VO}_{2}$ is sufficiently large to drive the program current of the memory unit for node dimension down to $1 \mathrm{~nm}$ (current density $\sim 10^{8} \mathrm{~A} / \mathrm{cm}^{2}$ ). However, the off-state current would be larger than $0.1 \mathrm{nA}$ with the node size larger than $1 \mathrm{~nm}$, giving rise to a too large sneak current over the entire array. Only $\mathrm{VO}_{2}$ selectors with node size $\sim 1 \mathrm{~nm}$ have high enough resistance in the insulating phase while simultaneously satisfying the criterion for $I_{\mathrm{ON}}$. The feasibility of such a design is still elusive as studies on devices approaching sub-10 nm are still in early stages.

The oN/OFF ratio of the selector must be at least four orders of magnitude $\left(R_{\mathrm{OFF}}>10^{4} R_{\mathrm{LRS}} \sim 10^{4} R_{\mathrm{ON}}\right)$. In practice, it is challenging to fabricate $\mathrm{VO}_{2}$ devices with ON/OFF ratio approaching the ideal case in cross-point fashion, as the grown $\mathrm{VO}_{2}$ films are typically nonstoichiometric on elemental metal electrodes. Experimental results of $I_{\mathrm{ON}}$ and $I_{\mathrm{OFF}}$ for $\mathrm{VO}_{2}$ cells with cell area smaller than 1 $\mu \mathrm{m}^{2}$ are shown as dots in Fig. 7(c). It can be clearly seen in these devices that the oN/OFF ratio is only around two orders of magnitude. How to grow high-quality $\mathrm{VO}_{2}$ thin films on a specific substrate while maintaining the transition magnitude that is close to bulk has become a key problem and will motivate new ideas for materials synthesis beyond tweaking deposition parameters.

From the above discussion, we can see that only certain $\mathrm{VO}_{2}$ devices with a specific cell area can fulfill the requirement for on and off current. On the other hand, if there were another material with a larger transition magnitude, the requirement on the cell size would be much less strict. One of the potential candidate materials is $\mathrm{NbO}_{2}$. Although the resistivity change of $\mathrm{NbO}_{2}$ right across the metal-toinsulator transition temperature is only two to three orders of magnitude, its resistivity can be more than $\sim 10^{4} \Omega \cdot \mathrm{cm}$ at room temperature and $\sim 10^{-3} \Omega \cdot \mathrm{cm}$ above the transition temperature $T_{C}(\sim 1070 \mathrm{~K})[154]$. This is due to its larger insulating bandgap compared with $\mathrm{VO}_{2}$ and much higher $T_{C}$. If the transition magnitude can be realized by a voltage bias, it would in theory offer a much larger design space, as shown in Fig. $7(\mathrm{c})$. Experimentally, $\mathrm{NbO}_{\mathrm{x}}$-based selector devices with high on current density $\left(>3 \times 10^{7} \mathrm{~A} / \mathrm{cm}^{2}\right)$ and high selectivity $\left(\sim 10^{4}\right)$ have been demonstrated [138], [139]. What is more, it was shown that the selector current path can be confined in a hybrid $\mathrm{NbO}_{2} / \mathrm{Nb}_{2} \mathrm{O}_{5}$ or $\mathrm{NbO}_{2} /$ $\mathrm{HfO}_{2}$ device and therefore reduce the effective device area [140]. As both ON and OFF switching speeds of a $110 \times 110 \mathrm{~nm}^{2}$ device are within $\sim 1-2 \mathrm{~ns}$ [7], the application of $\mathrm{NbO}_{2}$ selectors would be of significant interest if near-ideal selectivity could be demonstrated. Another advantage of $\mathrm{NbO}_{2}$-based selectors is that they could be more reliable than the $\mathrm{VO}_{2}$ counterparts, as the transition temperature is much higher than the typical CMOS junction temperature. A master list of correlated electron phase transitions with respective oN/OFF ratios can be found elsewhere [155].

When the oxides are nonstoichiometric, or stoichiometric but not in a specific crystallographic phase, they would not exhibit metal-to-insulator transitions and could not be utilized as selectors. However, bipolar nonvolatile switching could be observed in these oxides (for example, amorphous $\mathrm{VO}_{\mathrm{x}}$ deposited at room temperature [73], [74]). It is, therefore, possible to observe the coexistence of bipolar nonvolatile switching and threshold switching in inhomogeneous correlated materials [156], and such devices could even be self-selective, which may lead to simple one-element RRAM devices [157].

\section{B. Capacitive Memories}

Because the electronic phase transitions are often accompanied by the change in the dielectric properties [158]-[160] (from lattice and electronic change), Mott memories based on the change in capacitance have also been proposed. Fig. 8(a) shows the structure of a single

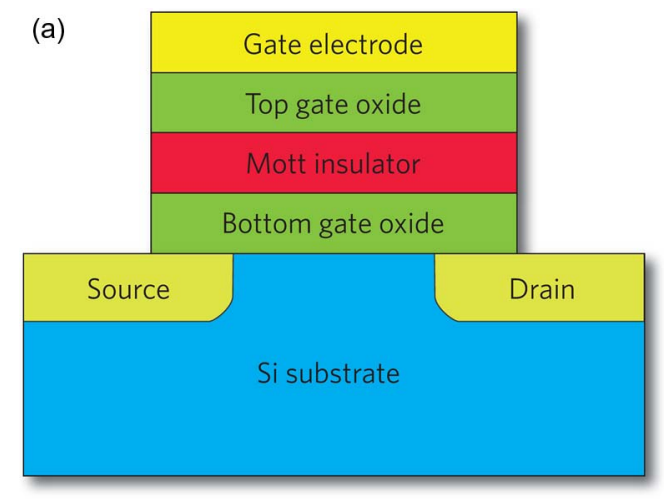

(b)

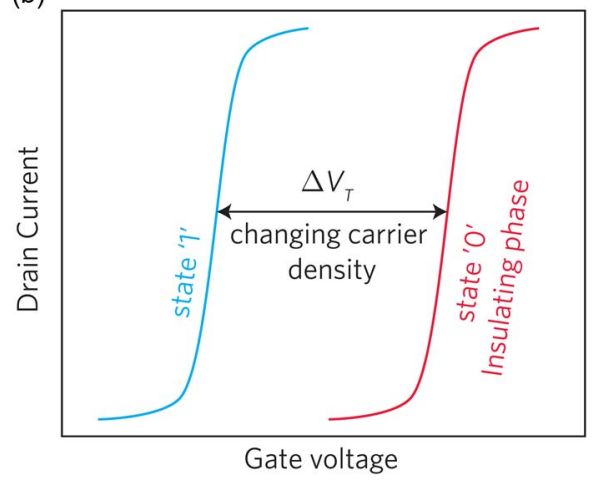

Fig. 8. (a) The structure of a single element phase transition memory. (b) The changes in free carrier density in the Mott material modifies the effective gate capacitance and shifts the threshold voltage of the field-effect transistor by $\Delta V_{T}$. (Panel (a) adapted with permission from [161].) 
element phase transition memory [161], [162], which is very similar to a Flash memory. A layer of Mott insulator is sandwiched between two gate oxide layers, which forms the gate oxide stack of a metal-oxide-semiconductor fieldeffect transistor (MOSFET). The effective gate capacitance would be different for insulating and metallic phases of the Mott insulator. In the ideal case, the effective gate capacitance is the series capacitance of three oxide layers when the sandwiched correlated oxide is in its insulating phase, and will only have contributions from the top and bottom gate oxide when the Mott insulator switches. This would lead to a drift in the threshold voltage of the MOSFET, as illustrated in Fig. 8(b). As a result, the state of the device can be read from the source-drain conductance under a specific gate voltage. Kim et al. showed that the MOSFET threshold voltage can be shifted by $0.5 \mathrm{~V}$ when using $\mathrm{VO}_{2}$ as the phase transition element [161]. Recently, it has been found that the gate electric field induces space charge polarization from ionized defects in a SNO-based memory device [89], [163]. It is interesting to note that the retention time after a gate pulse is on the order of $\sim 10 \mathrm{~s}$, longer than DRAM retention time, with a single transistor. Similarly, capacitance and inductance memory effects [164] enable the demonstration of memory metamaterials [165]-[168].

\section{Neuromorphic Devices: Neuristors}

In comparison with conventional digital computers, biological brains are massively parallel, faster, and more power efficient at certain tasks like image recognition and natural language processing [169]. There is growing interest in artificial neural circuits with the long-term goal of building computers mimicking the human brain and even helping to understand the brain function on a system level. The simulation of a brain at the software level is energy and space intensive [170]. As an example, a recent demonstration by Google of recognizing cats and human faces from video clips required 16000 cores and $\sim 100 \mathrm{~kW}$ of power [171]. Therefore, there is a resurgent trend in investigating the hardware-level implementation of neuromorphic computation [172]-[175]. The active area and power consumption are two primary concerns for hardware design. The implementation of neuromorphic computing on the basis of conventional complementary metal-oxidesemiconductor (CMOS), however, often requires a large numbers of transistors to simulate single neuron or synapse. For example, 147456 processors ( $10^{9} \mathrm{~B}$ per processor) and $10^{14} \mathrm{~B}$ of memory were used to simulate $10^{9}$ neurons and $\sim 10^{13}$ partially plastic synapses [172]. The power consumption was estimated at the level of hundreds of kilowatts [176]. The recent IBM TrueNorth chip used 5.4 billion transistors to mimic one million neurons and 256 million nonplastic synapses, with newly designed architecture [174]. To realize synaptic plasticity would require significantly more transistors. The inherent inefficiencies in simulating brains with CMOS are related to the digital versus analog way that CMOS circuits and the brain work, respectively. As a result, the question arises whether one can go below the architecture level and look for innovations at the materials or device level to use much fewer electronic devices to simulate the neurons and synapses. For example, RRAM may be used as a single device to mimic the synapse [175], [177]. The use of Mott insulators in neuromorphic devices is emerging in this context as an effective way to simulate neurons and synapses on a device level. In Sections III-C and III-D, we examine the motivation behind the use of correlated insulators in realizing electronic neurons and synapse, and discuss the related device performance and potential.

Neurons generate and transmit information through electrical and chemical signals. Each neuron possesses a body cell, which generates electrical spikes; an axon, which sends signals to other neurons; and dendrites, which receive signals. A synapse is a structure that permits the signal to transfer from the axon terminal of one cell and the dendrites of another, and its connection strength can be modulated, which is believed to lay the foundation of memories.

The electrical spiking signals in neurons, so called action potentials, are caused by the change in the ion concentrations (e.g., $\mathrm{Na}^{+}$and $\mathrm{K}^{+}$) within and outside the neuron cell body. The generation and propagation of action potentials relies on the pumping of the ions by voltage-gated ion channel and has been described by a set of nonlinear differential equations, that is the well-known HodgkinHuxley model [178]. Recently, it has been suggested by Pickett et al. [179] that the behavior of neurons initiating action potentials can be emulated by a neuristor that is built from two Mott threshold switches along with resistors and capacitors. Fig. 9(a) shows the circuit diagram of a neuristor. Two identical Mott switches, $M_{1}$ and $M_{2}$, together with two parallel capacitances $\left(C_{1}\right.$ and $\left.C_{2}\right)$, are biased by opposite dc bias to mimic the potassium and sodium ion channels in the Hodgkin-Huxley model. The input current charges the $C_{1}$ and $C_{2}$ capacitors before the voltage across $M_{1}$ and $M_{2}$ reaches their threshold voltage. When the threshold voltage is reached, the Mott insulator turns into metallic phase, leading to discharging of $C_{1}$ and $C_{2}$. The turning ON and OFF of the Mott switches is in analogy with the opening and closing of the ion channels, respectively. The offset between the charging/discharging time of the capacitors results in action potential voltage spikes, similar to biological systems. Fig. 9(b)-(e) shows the response of the circuit under two different stimulating input pulses. If the input voltage is superthreshold, the temporal profile of voltage output resembles that of biological action potentials and there is a maximum gain in the output voltage. On the other hand, when the input voltage is subthreshold, the output potential is weakened. Such response mimics the all-or-none law for neurons. The control of the interspike timing intervals and the spike width is achieved by modifying the capacitance value of $C_{1}$ and $C_{2}$. Fig. 9(f) shows various types of spiking behaviors achieved with different capacitance, indicating the versatility. It is worthwhile to 
(a)

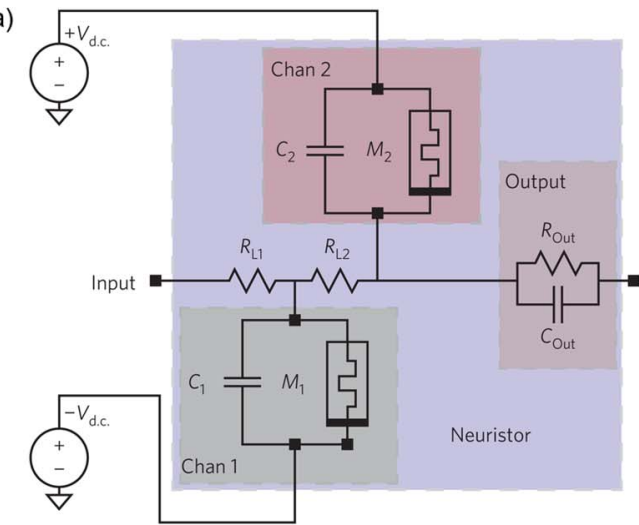

(b)

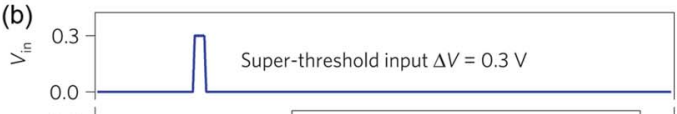

(c)

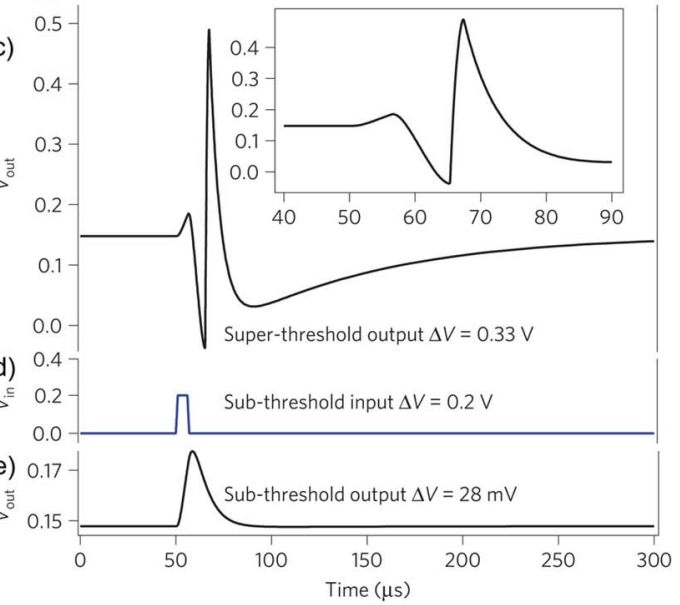

(f)

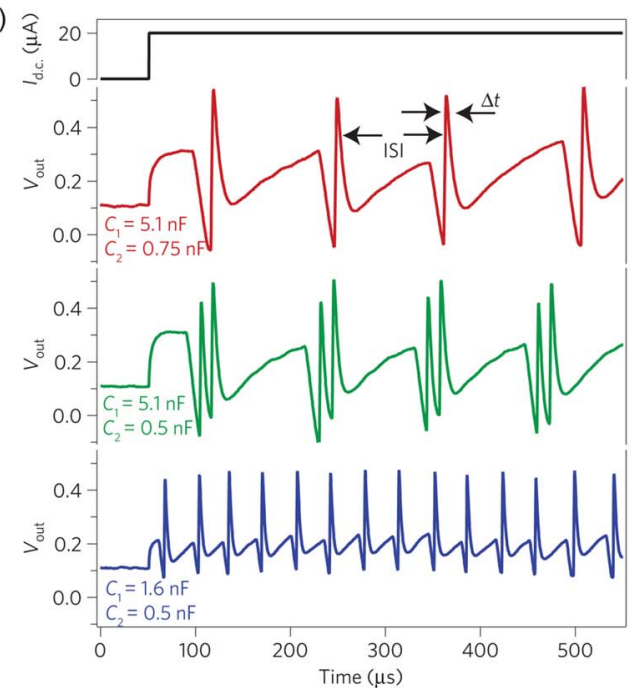

Fig. 9. (a) Circuit diagram of a scalable neuristor made with Mott switches and normal capacitors. $M_{1}$ and $M_{2}$ are two identical threshold-switching devices made of $\mathrm{NbO}_{2}$. (c) and (d) Superthreshold input and spiking-like output. (e) and (f) Subthreshold input and attenuated output. (g) Three types of spiking patterns: regular spiking, chattering, and fast spiking could be achieved in single circuit by adjusting the values of capacitors. (Adapted with permission from [179].) point out that neuristors have been demonstrated previously using other voltage-controlled negative differential resistance devices such as tunnel junctions [180], [181]. These designs usually require nonscalable inductors to operate [180], [181], while Mott switches can be scaled down to nanometer regime. Although the capacitors used were very large due to the large parasitic capacitance of the test apparatus, lowering the capacitance not only leads to reduced device area but also helps to generate higher frequency spikes and will be an important design criterion for more realistic circuits.

\section{Neuromorphic Devices: Synaptic Transistor}

As discussed above, neural cells can generate spikes under stimuli and the signal is passed on through synapses. Synaptic plasticity, the ability of synapses to strengthen or weaken, is believed to be the underlying mechanism of memory and learning. Spike-timing-dependent plasticity (STDP) is one where the synapse connection strength is adjusted based on the relative timing of a neuron's input (presynaptic) and output (postsynaptic) action potentials [182], [183]. Various types of STDP with different temporal dependency on spike timing have been observed. In the process of asymmetric STDP, the synaptic connection is strengthened if the presynaptic neuron fires before the postsynaptic neuron, and is weakened vice versa. The modification of strength is most significant if the input spikes occur immediately before/after the output spikes, as shown in Fig. 10(a). To emulate the synaptic function in electronic devices, the state (e.g., resistance) should be changed in a continuous fashion (analog) with reasonably long retention time (nonvolatile). Previously, artificial synapses have been implemented by two-terminal devices (for example, RRAM and PCRAM [175], [184]) and three-terminal FETs such as Si-based floating gate FET [185]. Recently, an ionic liquidgated synaptic transistor has been demonstrated [186]. The transistor structure is shown in Fig. 10(b). The source and the drain are analogous to preneuron and postneuron terminals, respectively. The channel material is a perovskite $\mathrm{SmNiO}_{3}$ (SNO), which exhibits a metal-to-insulator transition at $\sim 140{ }^{\circ} \mathrm{C}$ and its conductance $S$ emulates the strength of synapse. The channel SNO is gated by a drop of ionic liquid, and the spike timing difference is translated into a gate bias signal pulse with varying magnitude lasting the same period of time. Ionic liquid gating has been used widely in the condensed matter physics community to tune the carrier density in Mott insulators electrostatically. However, recent studies have shown that there are electrochemical processes occurring in these transistors, especially under large gate bias, which create defects and therefore modify the transport properties of the material [105], [106]. The persistent and continuous change in conductivity as a result of electrochemical processes is beneficial for the realization of synaptic plasticity. When a positive gate voltage is applied, oxygen vacancies are created inside SNO, resulting in an increase in the channel 
(a)

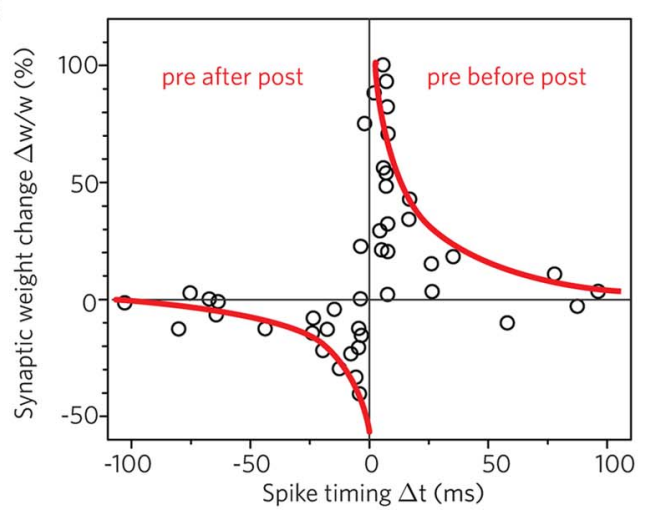

(b)

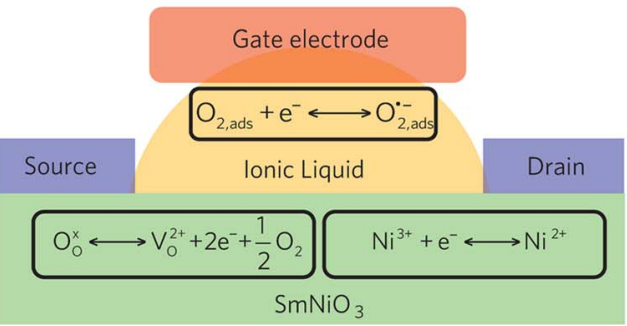

(c)

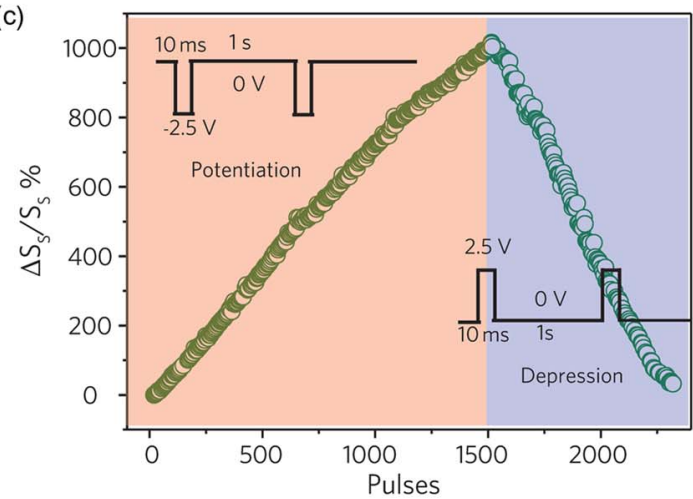

(d)

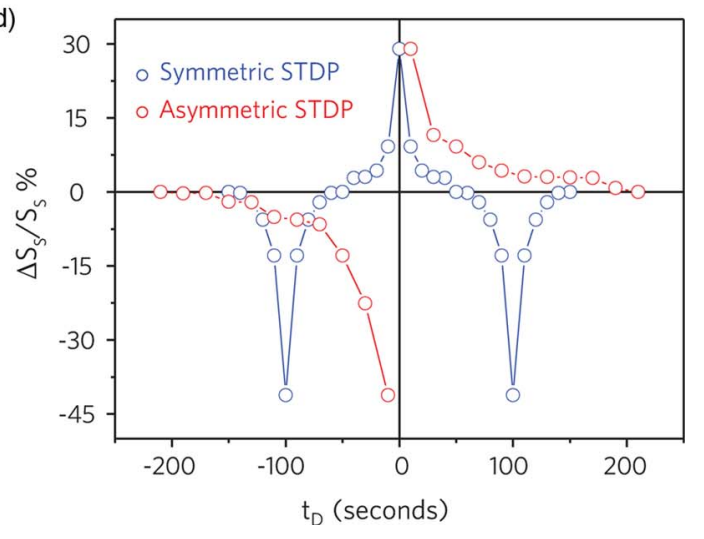

Fig. 10. (a) Asymmetric spike-timing-dependent plasticity in a biological synapse. The synapse weight is adjusted as a function of the relative time of presynaptic and postsynaptic spikings. (b) Device structure of a synaptic transistor based on $\mathrm{SmNiO}_{3}$, and the conductance modulation mechanism. (c) Sheet conductance $S_{s}$ modulation of $\mathrm{SmNiO}_{3}$ under potentiation and depression gate pulses. (d) Asymmetric and symmetric spike-timing-dependent plasticity demonstrated with a synaptic transistor. (Panel (a) adapted with permission from [183]; panels (b)-(d) adapted with permission from [186].) resistance. The overall defect reaction defect reaction in SNO can be written as $2 \mathrm{Ni}^{3+}+\mathrm{O}_{\mathrm{O}}^{x} \leftrightarrow 2 \mathrm{Ni}^{2+}+$ $\mathrm{V}_{\mathrm{O}}^{2+}+1 / 2 \mathrm{O}_{2}$. As discussed before, the electrical properties of Mott insulators in general are extremely sensitive to the carrier density and disorder, making them particularly suited. Fig. 10(c) shows the sheet conductance $S_{S}$ modulation of SNO under potentiation and depression gate pulses (periodic 10-ms gate pulses repeated once per second). The conductance can be programed accumulatively in an analog fashion similar to synapse. Both asymmetric and symmetric STDPs can be demonstrated using such synaptic transistors along with a neuron circuitry to convert spike time difference to gate voltage, as shown in Fig. 10(d). Recently, device-level classical conditioning and unlearning have been realized using SNO synaptic transistors [187].

Several aspects of performance of such synaptic transistors remain to be improved. First, the conductance modulation is relatively small. Future devices utilizing ions with higher mobility could enable more significant conductance change in synaptic transistors. Second, the demonstrated three-terminal synaptic transistors are slower compared with two-terminal RRAM- and PCRAM-based synapses, but the speed would scale down with the device dimension. Confining ionic liquids by microfluidic techniques should greatly help in studying the limits of switching such liquidoxide interfaces. What is more, the power/energy consumption of the electrochemical processes in these devices remains to be scrutinized. However, two-terminal synapses commonly operate with separated signal transmission and learning process, while these two processes occur simultaneously in biological synapses. Therefore, three-terminal synaptic transistors that are able to perform both operations concurrently may offer a promising path to efficient synapse emulation. In addition, the electrochemical transistor creates a defect diffusion profile at the channel surface, which could be better controlled than the stochastic defect creation in RRAM, and could therefore be more robust to device-to-device and cycle-to-cycle variations.

\section{KEY DEVICE PERFORMANCE METRICS}

\section{A. Limits of Switching Speed}

One of the most prominent features of Mott insulators is that the transition can be induced at fast timescales. As can be seen from Table 2, many electronic transitions reflected by the change in electric and optical conductivity can take place within $10^{2}-10^{3}$ fs when being triggered by optical excitations, comparable to transistor gate and interconnect delays. The experimental switching speed of voltage pulse-induced transitions is usually slower, on the order of a few nanoseconds, but still on par with RRAMs [145] and PCRAMs [188].

The optically induced transition dynamics of correlated insulators such as manganites [189], $\mathrm{V}_{2} \mathrm{O}_{3}$ [190], $\mathrm{VO}_{2}$ [4], 
Table 2 Metal-to-Insulator Transition Speed in Various Mott Insulators

\begin{tabular}{|c|c|c|c|c|}
\hline Material & Driving method & Probe & Speed & reference \\
\hline $\mathrm{Pr}_{0.7} \mathrm{Ca}_{0.3} \mathrm{MnO}_{3}$ & $\begin{array}{c}\text { Mid-IR } \\
\text { vibrational } \\
\text { excitation }\end{array}$ & Reflectivity & $1 \mathrm{ps}$ & [189] \\
\hline $\mathrm{Pr}_{0.7} \mathrm{Ca}_{0.3} \mathrm{MnO}_{3}$ & $\begin{array}{c}\text { Mid-IR } \\
\text { vibrational } \\
\text { excitation }\end{array}$ & $\begin{array}{l}\text { Electrical } \\
\text { conductivity }\end{array}$ & $4 \mathrm{~ns}$ & [189] \\
\hline $\mathrm{V}_{2} \mathrm{O}_{3}$ & Voltage pulse & $\begin{array}{l}\text { Electrical } \\
\text { conductivity }\end{array}$ & $\begin{array}{c}390 \\
\text { ps }\end{array}$ & [203] \\
\hline $\mathrm{V}_{2} \mathrm{O}_{3}$ & $\begin{array}{l}\text { Near-IR } \\
\text { excitation }\end{array}$ & $\begin{array}{c}\text { Far-IR } \\
\text { conductivity }\end{array}$ & $20 \mathrm{ps}$ & [190] \\
\hline $\mathrm{VO}_{2}$ & Voltage pulse & $\begin{array}{c}\text { Electric } \\
\text { conductivity }\end{array}$ & $2 \mathrm{~ns}$ & [6] \\
\hline $\mathrm{VO}_{2}$ & $\begin{array}{l}\text { Near-IR } \\
\text { excitation }\end{array}$ & Reflectivity & $75 \mathrm{fs}$ & [5] \\
\hline $\mathrm{VO}_{2}$ & $\begin{array}{c}\text { Near-IR } \\
\text { excitation }\end{array}$ & $\begin{array}{c}\text { Electron } \\
\text { diffraction }\end{array}$ & $300 \mathrm{fs}$ & [40] \\
\hline $\mathrm{NbO}_{2}$ & Voltage pulse & $\begin{array}{c}\text { Electric } \\
\text { conductivity }\end{array}$ & $\begin{array}{l}700 \\
\text { ps }\end{array}$ & [7] \\
\hline $1 T-\mathrm{TaS}_{2}$ & $\begin{array}{l}\text { Near-IR } \\
\text { excitation }\end{array}$ & $\begin{array}{c}\text { Time-resolved } \\
\text { ARPES }\end{array}$ & $100 \mathrm{fs}$ & [201] \\
\hline
\end{tabular}

[191]-[201], have been mostly studied by pump-probe techniques. In such experiments, a pump signal (typically with photon energy $\sim 1.5 \mathrm{eV}$ in the near-IR region, larger than the bandgap) creates enough number of electrons and holes in the conduction and valence band to collapse the gap, leading to nonthermal Mott transitions. A timedelayed probe is utilized to study various aspects of the transition dynamics. For example, an optical probe can detect the optical conductivity change, whereas ultrafast electron diffraction reveals structural transitions.

The interplay between different degrees of freedom is evident in ultrafast photoexcitation studies, which could potentially influence the speed of electronic phase transitions. Cavalleri et al. observed a transition speed bottle neck of $\sim 10^{2}$ fs in the reflectivity/transmission change of $\mathrm{VO}_{2}$ thin films after being optically excited, as shown in Fig. 11(a) [5]. The speed limit is attributed to the structural motion needed to collapse the bandgap. Baum et al. detected that the transition from stable insulating to metallic states will actually pass through a metastable phase [40]. The transition to the intermediate phase, characterized by local atomic motion and the dilation of the vanadiumvanadium bond, happens within $\sim 300 \mathrm{fs}$ and leads to the change in electrical conductivity. The slower relaxation from the intermediate to final tetragonal phase, manifested by the long-range atomic reordering and shear motion at sound wave velocity, takes a few picoseconds. This suggests that the electronic transition can occur simultaneously with local atomic motions, before the full crystallographic transition occurs.

The speed of voltage-triggered Mott transitions has been studied in both out-of-plane and in-plane two-terminal devices [6], [7], [64], [67], [202]-[206]. It is apparent that many factors such as material properties, device dimension, ambient temperature, and external electric field will in- fluence the transition speed. Regardless, the reported values of switching on time in $\mathrm{VO}_{2}, \mathrm{NbO}_{2}$, and other oxides range from $\sim 10^{-1}$ to $10^{1} \mathrm{~ns}$ [6], [7], [64], [67], [202]-[206]. Such speed is fast enough for many memory applications, considering that many of the reported values are still limited by parasitic capacitance and measurement instrumentation limitations [6], [205], [206]. Fig. 11(b) shows the current across a 400-nm-long $\mathrm{VO}_{2}$ two-terminal device as the voltage ramps up [6]. Within $2 \mathrm{~ns}$, the device resistance drops by two orders of magnitude, with similar magnitude as the thermal metal-to-insulator transition of the film.

The off time is an equally important parameter. Naturally, the device would become gradually easier to turn oN, but progressively more difficult to turn off with increasing ambient temperature, because of the decreasing free energy difference between the two phases. This leads to shorter switch on but longer switch off times, as can be predicted by a simple thermal model. Accordingly, there would be an optimized operation temperature range in terms of the device's overall speed, as illustrated in Fig. 11(c) [207]. It is desirable to select a region where the oN/OFF times are not significantly modified by chip temperature fluctuations as a result of device operation. The choice of operation temperature will also have an influence on the device reliability. With high transition temperature, it may require extra thermal isolation/dissipation structures to prevent intervention with nearby circuit elements. A too low transition temperature on the other hand may cause unwanted switching as a result of chip temperature fluctuation.

\section{B. Switching Energy of Phase Transitions}

One of the most important considerations for any practical memory device is their energy cost [208]. The minimum energy consumption per switching is given by the free energy difference between two phases at the operation temperature. 

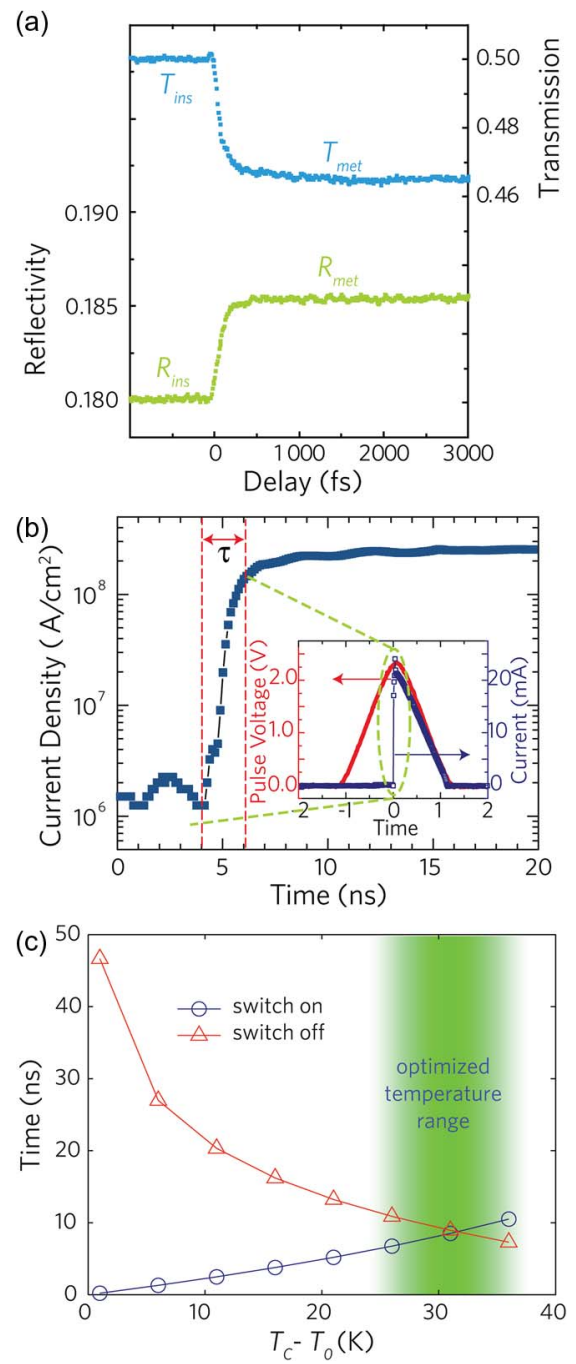

Fig. 11. (a) Ultrafast phase transition in $\mathrm{VO}_{2}$ as evidenced by reflection/transmission in optical pump-probe measurements. (b) Resistance change dynamics of a voltage-driven phase transition in $\mathrm{VO}_{2}$. The inset shows both the voltage and current temporal profiles. (c) on/ofr time as a function of operation temperature of a two-terminal Mott switch calculated by thermal modeling. (Panel (a) adapted with permission from [5]; panel (b) adapted with permission from [6]; panel (c) adapted with permission from [207].)

Table 3 lists the volumetric average heat capacity and latent heat for different materials [7], [209]-[211]. One obvious advantage of Mott memories is that the switching energy per bit will scale with the volume of the device and could be therefore drastically decreased to sub-10-nm size. For example, the switching energy of a $\mathrm{VO}_{2}$ or $\mathrm{NbO}_{2}$ cube with length of $10 \mathrm{~nm}$ is estimated to be around a few fJ ( $\sim 0.4$ fJ for $\mathrm{VO}_{2}$ and $\sim 2$ fJ for $\mathrm{NbO}_{2}$ ), as estimated from the parameters in Table 3. It could also be seen that the energy cost to switch stoichiometric $\mathrm{NbO}_{2}$ is larger than that of the same volume of stoichiometric $\mathrm{VO}_{2}$. However, the heat capacity, phase transition latent heat, and transition temperature will also depend on material stoichiom- etry and defects. Thus, engineering techniques to reduce the energy per volume could be important to realize energy-efficient devices with large on/OFF ratio.

\section{Reliability}

Reliability is one of the key concerns for Mott twoterminal switches especially if Joule heating plays an important role. Variations in the oN/OFF resistivity value are rather small, as shown in Fig. 12(a). The resistance of a single device can be changed by choosing the proper length and area. On the other hand, the initial E-MIT switching process could be stochastic as reflected in the variations in the $V_{\text {th }}$ in Fig. 12(b). The initial partial switching could happen in different regions of the device as evidenced in optical microscopy studies. Reducing the device size to material grain size could be helpful to reduce such variations. On the other hand, there are usually less variations in the $V_{\mathrm{th}}^{*}$, as the turnoff process is determined by the power input and heat dissipation in the device.

The variations between devices are related to the nonuniformity of the prepared thin films. Growth and processing techniques need to be optimized to achieve uniformity in the transition properties. In some cases, an electroforming process needs to be performed before the device could be switched on [59], which is possibly due to interfacial oxide layer formed at the metal/insulator interface and does not occur in all devices. From reliability point of view, devices without the need of electroforming are more desired, which requires proper selection of contact electrode materials. As for endurance, Radu et al. demonstrated that $\mathrm{VO}_{2}$ switches have endurance better than $10^{9}$ cycles [135]. Similar endurance performance has been demonstrated in $\mathrm{NbO}_{2}$ [7]. Thin films confined to the substrate are more robust to the stress relaxation phenomena and this is in sharp contrast with bulk single crystals where the crystals shatter after one to few thermal cycles as a result of the structural transition.

\section{D. (Non)Volatility of State}

Devices based on correlated oxides can be either volatile or nonvolatile depending on their operation principles. The switching is volatile for voltage triggered $\mathrm{MIT}$ in $\mathrm{NbO}_{2}$ and $\mathrm{VO}_{2}$, because the transitions are either induced by injecting extra carriers or Joule heating (also referred to as threshold switches). For both cases, the metallic phase cannot be sustained once external driving force is removed. On the other hand, if the MIT is driven by carriers that are created (or annihilated) from defect density changes as in the synaptic transistor discussed above, the device will operate in a nonvolatile fashion, since the defects persist once created. Both volatile and nonvolatile behaviors have been demonstrated, and one can choose the desired operation principle/properties based on the applications. For neuromorphic circuits, the synapses need to be nonvolatile whereas the neurons are composed of volatile elements. In nickelate synaptic transistors for instance, due to low 
Table 3 Volumetric Free Energy Cost for Phase Transitions

\begin{tabular}{|c|c|c|c|c|c|}
\hline Material & $\begin{array}{c}\text { Volumetric } \\
\text { heat capacity } \\
\mathrm{a}, \mathrm{b}\end{array}$ & $\begin{array}{c}\text { Volumetric } \\
\text { enthalpy of } \\
\text { transformation }\end{array}$ & $\begin{array}{c}\text { Transition } \\
\text { temperature } \mathrm{b}\end{array}$ & $\begin{array}{c}\text { Total } \\
\text { volumetric } \\
\text { enthalpy }\end{array}$ & Reference \\
\hline $\mathrm{NbO}_{2}$ & $\begin{array}{c}2.6 \times 10^{6} \mathrm{~J} \mathrm{~m}^{-3} \\
\mathrm{~K}^{-1}\end{array}$ & $\begin{array}{c}1.6 \times 10^{8} \mathrm{~J} \\
\mathrm{~m}^{-3}\end{array}$ & $1070 \mathrm{~K}$ & $\begin{array}{c}2.2 \times 10^{9} \\
\mathrm{~J} \mathrm{~m}^{-3}\end{array}$ & {$[7,209]$} \\
\hline $\mathrm{VO}_{2}$ & $\begin{array}{c}3.0 \times 10^{6} \mathrm{~J} \mathrm{~m}^{-3} \\
\mathrm{~K}^{-1}\end{array}$ & $\begin{array}{c}2.4 \times 10^{8} \mathrm{~J} \\
\mathrm{~m}^{-3}\end{array}$ & $340 \mathrm{~K}$ & $\begin{array}{c}3.6 \times 10^{8} \\
\mathrm{~J} \mathrm{~m}^{-3}\end{array}$ & $\begin{array}{c}{[210,} \\
211]\end{array}$ \\
\hline \multicolumn{5}{|c|}{ a Heat capacity value is temperature dependent and taken as the average from room } \\
temperature to the transition temperature. \\
b The given numbers serve as references and only apply to stoichiometric transition metal \\
oxides with specific crystal structure. The exact values of these physical constants depend \\
on the sample's stoichiometry and can be tuned over a wide range. \\
\hline
\end{tabular}

diffusivity of defects in solids at room temperature, the channel resistance does not degrade even after a few months. The retention properties of these devices would certainly be interesting for future studies.

\section{OUTLOOK AND FUTURE RESEARCH NEEDS}

Orbitronic memory devices that utilize collective state switching are being actively explored in the materials sciences and device community. Many device parameters, including switching voltage and speed, are compatible with RRAMs and PCRAMs to form 1S1R nonvolatile memory arrays, while the oN/OFf ratio still needs to be improved. Thermal stability of phase change switches in highly integrated circuits requires attention. Radiation effects on cor-
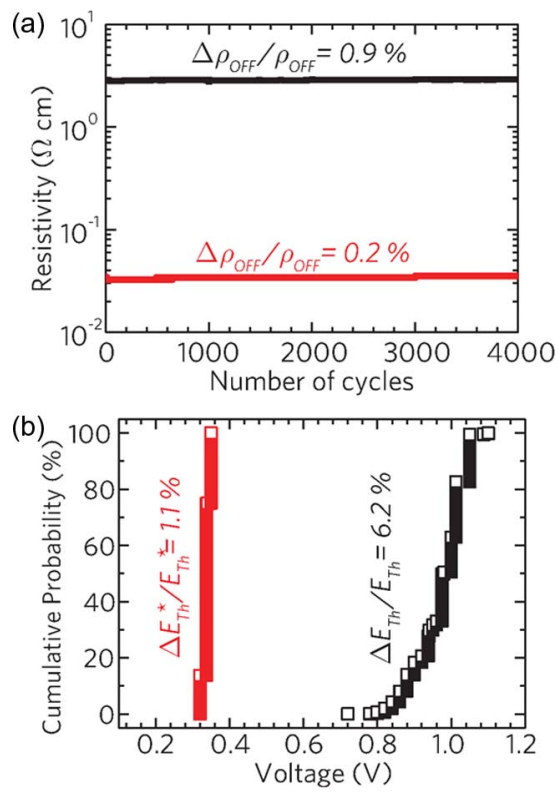

Fig. 12. Variation of (a) on/orf resistance and (b) threshold voltage of a $\mathrm{VO}_{2}$ two-terminal device over a few thousand cycles. related insulators and device reliability is another area that calls for much emphasis. Device physics models that can capture correlation effects even to an approximate level therefore are essential. While first principle studies can provide critical insights into many aspects of the materials, often they cannot capture the whole spectrum of complex phenomena in real materials. Mott materials are of interest in capacitive memories in Flash-like structures. The application of Mott insulators in neuromorphic devices is in early stages, and it may be possible to monolithically integrate such neural elements with conventional Si devices to form brain-inspired circuits. The ability to continuously tune the electrical resistance of Mott insulators, timedependent resistance modulation as well as obtain high nonlinearity positions them uniquely in such circuits.

At the same time, many important questions remain. The underlying physical mechanisms of MIT under various excitation sources need to be studied. Whether the final electronic states are identical regardless of the excitation source requires combined in situ probes. Many of the existing studies have been performed on vanadium dioxide partly due to the proximity of the transition to room temperature and ease of applying various experimental techniques. For materials showing transitions at higher temperature such as nickelates and niobium oxides, there is still plenty to be understood. New instrumentation will be needed to perform even the most basic in situ experiments due to the environmental (oxygen partial pressure) control needed to maintain the phase and compositional stability. Modeling the scaling behavior of memory devices requires deeper understanding of the electronic band structures of Mott insulators. High-quality heterojunctions or homo p-n junctions are certainly helpful to understand basic electrostatics. The electrical properties of interfaces between Mott insulators and band insulators (i.e., gate dielectric)/metals (i.e., contacts) become increasingly important as devices scale down, which we know very little of. In turn, this implies that we need to be able to control defects at the oxide interfaces to a minimum to probe the intrinsic properties. Dispersive capacitance in such complex oxides often poses a challenge in applying commonly used 
capacitance-voltage curve analysis to understand electrical nature of gate stack interfaces.

From a materials perspective, the growth of Mott insulators has been challenging because of defects and nonstoichiometry related degradation of the transition magnitude (i.e., on/off ratio). While epitaxial growth of oxides on lattice-matched single crystal substrates is mature, growth of phase-pure films on nonlattice matched substrates such as metals and atomic layer deposited oxides in a CMOS- compatible process is an area that requires intense effort. On the other hand, controlled defect creation by electric fields modifies orbital occupancy of correlated electrons and induces nonvolatile resistance changes. Exploiting the great sensitivity of their electrical properties to defects could, therefore, lead to novel applications in ionic-electronic devices. Collaborative efforts between material growers and device engineers will advance the frontiers of this field in the coming decades and will foster the growth of orbitronics.

\section{REFERENCES}

[1] J. H. de Boer and E. J. W. Verwey, "Semi-conductors with partially and with completely filled 3D-lattice bands," Proc. Phys. Soc., vol. 49, p. 59, 1937.

[2] N. F. Mott, "The basis of the electron theory of metals, with special reference to the transition metals," Proc. Phys. Soc. A, vol. 62, p. 416, 1949.

[3] N. Mott, "On metal-insulator transitions," J. Solid State Chem., vol. 88, pp. 5-7, 1990.

[4] A. Cavalleri et al., "Femtosecond structural dynamics in $\mathrm{VO}_{2}$ during an ultrafast solid-solid phase transition," Phys. Rev. Lett., vol. 87, 2001, Art. ID. 237401.

[5] A. Cavalleri, T. Dekorsy, H. H. W. Chong, J. C. Kieffer, and R. W. Schoenlein, "Evidence for a structurally-driven insulator-to-metal transition in $\mathrm{VO}_{2}$ : A view from the ultrafast timescale," Phys. Rev. B, vol. 70, 2004, Art. ID. 161102.

[6] Y. Zhou et al., "Voltage-triggered ultrafast phase transition in vanadium dioxide switches," IEEE Electron Device Lett., vol. 34, no. 2, pp. 220-222, Feb. 2013.

[7] M. D. Pickett and R. S. Williams, "Sub-100 fJ and sub-nanosecond thermally driven threshold switching in niobium oxide crosspoint nanodevices," Nanotechnology, vol. 23, 2012, Art. ID. 215202.

[8] International Technology Roadmap for Semiconductors, 2013. [Online]. Available: http://www.itrs.net/Links/2013ITRS/ Summary2013.htm

[9] Z. Yang, C. Ko, and S. Ramanathan, "Oxide electronics utilizing ultrafast metal-insulator transitions," Annu. Rev. Mater. Res., vol. 41, pp. 337-367, 2011.

[10] M. M. Qazilbash et al., "Mott transition in $\mathrm{VO}_{2}$ revealed by infrared spectroscopy and nano-imaging," Science, vol. 318, pp. 1750-1753, 2007.

[11] J. Wei, Z. Wang, W. Chen, and D. H. Cobden, "New aspects of the metal-insulator transition in single-domain vanadium dioxide nanobeams," Nature Nanotechnol., vol. 4, pp. 420-424, 2009.

[12] J. Cao et al., "Strain engineering and one-dimensional organization of metal-insulator domains in single-crystal vanadium dioxide beams," Nature Nanotechnol., vol. 4, pp. 732-737, 2009.

[13] I. I. Mazin et al., "Charge ordering as alternative to Jahn-Teller distortion," Phys. Rev. Lett., vol. 98, 2007, Art. ID. 176406.

[14] E. Verwey, "Electronic conduction of magnetite $\left(\mathrm{Fe}_{3} \mathrm{O}_{4}\right)$ and its transition point at low temperatures," Nature, vol. 144, pp. 327-328, 1939.

[15] J. A. Alonso et al., "High-temperature structural evolution of $\mathrm{RNiO}_{3}$
( $\mathrm{R}=\mathrm{Ho}, \mathrm{Y}, \mathrm{Er}, \mathrm{Lu})$ perovskites: Charge disproportionation and electronic localization," Phys. Rev. B, vol. 64, 2001, Art. ID. 094102.

[16] M. Imada, A. Fujimori, and Y. Tokura, "Metal-insulator transitions," Rev. Modern Phys., vol. 70, pp. 1039-1263, 1998.

[17] J. M. Rondinelli, S. J. May, and J. W. Freeland, "Control of octahedral connectivity in perovskite oxide heterostructures: An emerging route to multifunctional materials discovery," MRS Bull., vol. 37, pp. 261-270, 2012.

[18] J. B. Goodenough, "Electronic and ionic transport properties and other physical aspects of perovskites," Rep. Progr. Phys., vol. 67, p. $1915,2004$.

[19] N. F. Mott and L. Friedman "Metal-insulator transitions in $\mathrm{VO}_{2}$, Ti2O3 and $\mathrm{Ti}_{2-\mathrm{x}} \mathrm{V}_{\mathrm{x}} \mathrm{O}_{3}$," Philosoph. Mag., vol. 30, pp. 389-402, 1974.

[20] A. P. Ramirez, "Colossal magnetoresistance," J. Phys., Condensed Matter, vol. 9, pp. 81718199, 1997.

[21] S. M. Wu et al., "Reversible electric control of exchange bias in a multiferroic field-effect device," Nature Mater., vol. 9, pp. 756-761, 2010.

[22] S. Fusil, V. Garcia, A. Barthélémy, and M. Bibes, "Magnetoelectric devices for spintronics," Annu. Rev. Mater. Res., vol. 44, pp. 91-116, 2014.

[23] A. I. Khan et al., "Negative capacitance in a ferroelectric capacitor," Nature Mater. vol. 14, pp. 182-186, 2015.

[24] J. B. Goodenough, "The two components of the crystallographic transition in $\mathrm{VO}_{2}$," J. Solid State Chem., vol. 3, pp. 490-500, 1971.

[25] A. Zylbersztejn and N. F. Mott, "Metal-insulator transition in vanadium dioxide," Phys. Rev. B, vol. 11, pp. 4383-4395, 1975.

[26] R. M. Wentzcovitch, W. W. Schulz, and P. B. Allen, " $\mathrm{VO}_{2}$ : Peierls or Mott-Hubbard? A view from band theory," Phys. Rev. Lett., vol. 72, pp. 3389-3392, 1994.

[27] V. Eyert, " $\mathrm{VO}_{2}$ : A novel view from band theory," Phys. Rev. Lett., vol. 107, 2011, Art. ID. 016401

[28] T. M. Rice, H. Launois, and J. P. Pouget, "Comment on $\mathrm{VO}_{2}$ : Peierls or Mott-Hubbard? A view from Band theory," Phys. Rev. Lett., vol. 73, pp. 3042-3042, 1994.

[29] R. Eguchi et al., "Photoemission evidence for a Mott-Hubbard metal-insulator transition in $\mathrm{VO}_{2}$," Phys. Rev. B, vol. 78 2008, Art. ID. 075115

[30] M. W. Haverkort et al., "Orbital-assisted metal-insulator transition in $\mathrm{VO}_{2}$," Phys. Rev. Lett., vol. 95, 2005, Art. ID. 196404.
[31] J. P. Pouget et al., "Dimerization of a linear Heisenberg chain in the insulating phases of $\mathrm{V}_{1-\mathrm{x}} \mathrm{Cr}_{\mathrm{x}} \mathrm{O}_{2}$," Phys. Rev. B, vol. 10, pp. 1801-1815, 1974.

[32] J. P. Pouget, H. Launois, J. P. D’Haenens, P. Merenda, and T. M. Rice, "Electron localization induced by uniaxial stress in pure $\mathrm{VO}_{2}$," Phys. Rev. Lett., vol. 35, pp. 873-875, 1975.

[33] S. Biermann, A. Poteryaev, A. I. Lichtenstein, and A. Georges, "Dynamical singlets and correlation-assisted peierls transition in $\mathrm{VO}_{2}, "$ Phys. Rev. Lett., vol. 94, 2005, Art. ID. 026404

[34] A. Liebsch, H. Ishida, and G. Bihlmayer, "Coulomb correlations and orbital polarization in the metal-insulator transition of $\mathrm{VO}_{2}$," Phys. Rev. B, vol. 71, 2005, Art. ID. 085109.

[35] J. M. Tomczak, F. Aryasetiawan, and S. Biermann, "Effective bandstructure in the insulating phase versus strong dynamica correlations in metallic $\mathrm{VO}_{2}$," Phys. Rev. B, vol. 78, 2008, Art. ID. 115103.

[36] T. C. Koethe et al., "Transfer of spectral weight and symmetry across the metal-insulator transition in $\mathrm{VO}_{2}$," Phys. Rev. Lett., vol. 97, 2006, Art. ID. 116402.

[37] A. S. Belozerov, M. A. Korotin, V. I. Anisimov, and A. I. Poteryaev, "Monoclinic M1 phase of $\mathrm{VO}_{2}$ : Mott-Hubbard versus band insulator," Phys. Rev. B, vol. 85, 2012, Art. ID. 045109.

[38] S. Kim, K. Kim, C.-J. Kang, and B. I. Min, "Correlation-assisted phonon softening and the orbital-selective Peierls transition in $\mathrm{VO}_{2}$," Phys. Rev. B, vol. 87, 2013, Art. ID. 195106.

[39] J. D. Budai et al., "Metallization of vanadium dioxide driven by large phonon entropy," Nature, vol. 515, pp. 535-539, 2014.

[40] P. Baum, D.-S. Yang, and A. H. Zewail, "4D visualization of transitional structures in phase transformations by electron diffraction," Science, vol. 318, pp. 788-792, 2007.

[41] H.-T. Kim et al., "Monoclinic and correlated metal phase $\mathrm{inVO}_{2}$ as evidence of the Mott transition: Coherent phonon analysis," Phys. Rev. Lett., vol. 97, 2006, Art. ID. 266401.

[42] E. Arcangeletti et al., "Evidence of a pressure-induced metallization process in monoclinic $\mathrm{VO}_{2}$," Phys. Rev. Lett., vol. 98, 2007, Art. ID. 196406.

[43] B.-J. Kim et al., "Micrometer x-ray diffraction study of $\mathrm{VO}_{2}$ films: Separation between metal-insulator transition and structural phase transition," Phys. Rev. B, vol. 77, 2008, Art. ID. 235401.

[44] K. L. Holman et al., "Insulator to correlated metal transition in $\mathrm{V}_{1-\mathrm{x}} \mathrm{Mo}_{\mathrm{x}} \mathrm{O}_{2}$," Phys. Rev. B, vol. 79, 2009, Art. ID. 245114. 
[45] Z. Tao et al., "Decoupling of structural and electronic phase transitions in $\mathrm{VO}_{2}$," Phys. Rev. Lett., vol. 109, 2012, Art. ID. 166406.

[46] J. Laverock et al., "Direct observation of decoupled structural and electronic transitions and an ambient pressure monocliniclike metallic phase of $\mathrm{VO}_{2}$," Phys. Rev. Lett., vol. 113, 2014, Art. ID. 216402.

[47] W.-P. Hsieh et al., "Evidence for photo-induced monoclinic metallic $\mathrm{VO}_{2}$ under high pressure," Appl. Phys. Lett., vol. 104, 2014, Art. ID. 021917.

[48] J. B. Hatch et al., "Intermediate metallic phase in $\mathrm{VO}_{2}$ observed with scanning tunneling spectroscopy," Phys. Chem. Chem. Phys., vol. 16, pp. 14183-14188, 2014.

[49] D. Wegkamp et al., "Instantaneous band gap collapse in photoexcited monoclinic $\mathrm{VO}_{2}$ due to photocarrier doping," Phys. Rev. Lett., vol. 113, 2014, Art. ID. 216401.

[50] S. Shin et al., "Vacuum-ultraviolet reflectance and photoemission study of the metal-insulator phase transitions in $\mathrm{VO}_{2}, \mathrm{~V}_{6} \mathrm{O}_{13}$, and $\mathrm{V}_{2} \mathrm{O}_{3}$," Phys. Rev. B, vol. 41, pp. 4993-5009, 1990.

[51] S. A. Corr, D. P. Shoemaker, B. C. Melot, and R. Seshadri, "Real-space investigation of structural changes at the metal-insulator transition in $\mathrm{VO}_{2}, "$ Phys. Rev. Lett., vol. 105, 2010, Art. ID. 056404.

[52] H. H. Kung, Transition Metal Oxides: Surface Chemistry and Catalysis. Amsterdam, The Netherlands: Elsevier, 1989.

[53] N. F. Quackenbush et al., "Nature of the metal insulator transition in ultrathin epitaxial vanadium dioxide," Nano Lett., vol. 13, pp. 4857-4861, 2013.

[54] J. I. Sohn et al., "Surface-stress-induced mott transition and nature of associated spatial phase transition in single crystalline $\mathrm{VO}_{2}$ nanowires," Nano Lett., vol. 9, pp. 3392-3397, 2009.

[55] R. G. Moore et al., "A surface-tailored, purely electronic, Mott metal-to-insulator transition," Science, vol. 318, pp. 615-619, 2007.

[56] O. Friedt, "Structural and magnetic aspects of the metal-insulator transition in $\mathrm{Ca}_{2-\mathrm{x}} \mathrm{Sr}_{\mathrm{x}} \mathrm{RuO}_{4}$," Phys. Rev. B, vol. 63 , 2001, Art. ID. 174432.

[57] S. Nakatsuji et al., "Heavy-mass Fermi liquid near a ferromagnetic instability in layered ruthenates," Phys. Rev. Lett., vol. 90, 2003, Art. ID. 137202.

[58] S. Nakatsuji and Y. Maeno, "Quasi-two-dimensional Mott transition system $\mathrm{Ca}_{2-x} \mathrm{Sr}_{\mathrm{x}} \mathrm{RuO}_{4}$," Phys. Rev. Lett., vol. 84, pp. 2666-2669, 2000.

[59] F. A. Chudnovskii, L. L. Odynets, A. L. Pergament, and G. B. Stefanovich, "Electroforming and switching in oxides of transition metals: The role of metal-insulator transition in the switching mechanism," $J$ Solid State Chem., vol. 122, pp. 95-99, 1996.

[60] F. Chudnovskii, A. Pergament, G. Stefanovich, P. Metcalf, and J. Honig, "Switching phenomena in chromium-doped vanadium sesquioxide," J. Appl. Phys., vol. 84, pp. 2643-2646, 1998

[61] A. Asamitsu, Y. Tomioka, H. Kuwahara, and Y. Tokura, "Current switching of resistive states in magnetoresistive manganites," Nature, vol. 388, pp. 50-52, 1997.

[62] S. Lee et al., "Electrically driven phase transition in magnetite nanostructures," Nature Mater., vol. 7, pp. 130-133, 2007.

[63] S. Yamanouchi, Y. Taguchi, and Y. Tokura, "Dielectric breakdown of the insulating charge-ordered state in $\mathrm{La}_{2-\mathrm{x}} \mathrm{Sr}_{\mathrm{x}} \mathrm{NiO}_{4}$," Phys. Rev. Lett., vol. 83, pp. 5555-5558, 1999.

[64] J. Leroy et al., "High-speed metal-insulator transition in vanadium dioxide films induced by an electrical pulsed voltage over nano-gap electrodes," Appl. Phys. Lett., vol. 100, 2012, Art. ID. 213507.

[65] K. L. Chopra, "Current-controlled negative resistance in thin niobium oxide films," Proc. IEEE, vol. 51, no. 6, pp. 941-942, Jun. 1963.

[66] D. V. Geppert, "A new negative-resistance device," Proc. IEEE, vol. 51, no. 1 , pp. 223-223, Jan. 1963.

[67] S. D. Ha, Y. Zhou, C. J. Fisher, S. Ramanathan, and J. P. Treadway, "Electrical switching dynamics and broadband microwave characteristics of $\mathrm{VO}_{2}$ radio frequency devices," J. Appl. Phys., vol. 113, 2013, Art. ID. 184501.

[68] R. Waser and M. Aono, "Nanoionics-based resistive switching memories," Nature Mater., vol. 6, pp. 833-840, 2007.

[69] C. N. Berglund, "Thermal filaments in vanadium dioxide," IEEE Trans. Electron Devices, vol. 16, no. 5, pp. 432-437, May 1969.

[70] J. Sakai and M. Kurisu, "Effect of pressure on the electric-field-induced resistance switching of $\mathrm{VO}_{2}$ planar-type junctions," Phys. Rev. B, vol. 78, 2008, Art. ID. 033106

[71] J. Duchene, M. Terraillon, P. Pailly, and G. Adam, "Filamentary conduction in $\mathrm{VO}_{2}$ coplanar thin-film devices," Appl. Phys. Lett., vol. 19, pp. 115-117, 1971.

[72] L. Pellegrino et al., "Multistate memory devices based on free-standing $\mathrm{VO}_{2} / \mathrm{TiO}_{2}$ microstructures driven by Joule self-heating," Adv. Mater., vol. 24, pp. 2929-2934, 2012.

[73] M. Son et al., "Self-selective characteristics of nanoscaleVO $\mathrm{x}_{\mathrm{x}}$ devices for high-density ReRAM applications," IEEE Electron Device Lett., vol. 33, no. 5, pp. 718-720, May 2012.

[74] F. J. Wong, T. S. Sriram, B. R. Smith, and S. Ramanathan, "Bipolar resistive switching in room temperature grown disordered vanadium oxide thin-film devices," Solid-State Electron., vol. 87, pp. 21-26, 2013.

[75] A. V. Pohm, C. Sie, R. Uttecht, V. Kao, and O. Agrawal, "Chalcogenide glass bistable resistivity (Ovonic) memories," IEEE Trans. Magn., vol. 6, no. 3, pp. 592-592, Sep. 1970.

[76] M. Wuttig and N. Yamada, "Phase-change materials for rewriteable data storage," Nature Mater., vol. 6, pp. 824-832, 2007.

[77] T. Oka and N. Nagaosa, "Interfaces of correlated electron systems: Proposed mechanism for colossal electroresistance," Phys. Rev. Lett., vol. 95, 2005, Art. ID. 266403.

[78] R. Fors, S. I. Khartsev, and A. M. Grishin, "Giant resistance switching in metal-insulator-manganite junctions: Evidence for Mott transition," Phys. Rev. B, vol. 71, 2005, Art. ID. 045305.

[79] P. P. Boriskov, A. A. Velichko, A. L. Pergament, G. B. Stefanovich, and D. G. Stefanovich, "The effect of electric field on metal-insulator phase transition in vanadium dioxide," Tech. Phys. Lett., vol. 28, pp. 406-408, 2002.

[80] C. Ko and S. Ramanathan, "Observation of electric field-assisted phase transition in thin film vanadium oxide in a metal-oxide-semiconductor device geometry," Appl. Phys. Lett., vol. 93, 2008, Art. ID. 252101.

[81] G. Stefanovich, A. Pergament, and D. Stefanovich, "Electrical switching and Mott transition in $\mathrm{VO}_{2}$," J. Phys., Condensed Matter, vol. 12, pp. 8837-8845 2000.

[82] P. Stoliar et al., "Universal electric-field-driven resistive transition in narrow-gap Mott insulators," Adv. Mater., vol. 25, pp. 3222-3226, 2013.

[83] F. Nakamura et al., "Electric-field-induced metal maintained by current of the Mott insulator $\mathrm{Ca}_{2} \mathrm{RuO}_{4}$," Sci. Rep., vol. 3, 2013, Art. ID. 2536.

[84] A. Zimmers et al., "Role of thermal heating on the voltage induced insulator-metal transition in $\mathrm{VO}_{2}$," Phys. Rev. Lett., vol. 110, 2013, Art. ID. 056601.

[85] X. Zhong, X. Zhang, A. Gupta, and P. LeClair, "Avalanche breakdown in microscale $\mathrm{VO}_{2}$ structures," J. Appl. Phys., vol. 110, 2011, Art. ID. 084516

[86] G. Gopalakrishnan, D. Ruzmetov, and S. Ramanathan, "On the triggering mechanism for the metal-insulator transition in thin film $\mathrm{VO}_{2}$ devices: Electric field versus thermal effects," J. Mater. Sci., vol. 44, pp. 5345-5353, 2009.

[87] B. S. Mun et al., "Role of joule heating effect and bulk-surface phases in voltage-driven metal-insulator transition in $\mathrm{VO}_{2}$ crystal," Appl. Phys. Lett., vol. 103, 2013, Art. ID. 061902.

[88] B. Fisher, J. Genossar, K. B. Chashka, L. Patlagan, and G. M. Reisner, "Metal-insulator transition upon heating and negative-differential-resistive-switching induced by self-heating in $\mathrm{BaCo}_{0.9} \mathrm{Ni}_{0.1} \mathrm{~S}_{1.8}$, Appl. Phys. Lett., vol. 104, 2014, Art. ID. 153511

[89] L. Cario, C. Vaju, B. Corraze, V. Guiot, and E. Janod, "Electric-field-induced resistive switching in a family of Mott insulators: Towards a new class of RRAM memories," Adv. Mater., vol. 22, pp. 5193-5197, 2010.

[90] P. Stoliar et al., "Universa electric-field-driven resistive transition in narrow-gap Mott insulators," Adv. Mater., vol. 25, pp. 3222-3226, 2013.

[91] V. Dubost et al., "Resistive switching at the nanoscale in the Mott insulator compound $\mathrm{GaTa}_{4} \mathrm{Se}_{8}$," Nano Lett., vol. 13, pp. 3648-3653, 2013.

[92] Y. Zhou and S. Ramanathan, "Correlated electron materials and field effect transistors for logic: A review," Critical Rev. Solid State Mater. Sci., vol. 38, pp. 286-317, 2013.

[93] K. Hyun-Tak et al., "Mechanism and observation of Mott transition in $\mathrm{VO}_{2}$-based two- and three-terminal devices," New J. Phys., vol. 6, 2004, Art. ID. 52.

[94] S. Sengupta et al., "Field-effect modulation of conductance in $\mathrm{VO}_{2}$ nanobeam transistors withHfO $\mathrm{H}_{2}$ as the gate dielectric," Appl. Phys. Lett., vol. 99, 2011, Art. ID. 062114.

[95] D. M. Newns et al., "Mott transition field effect transistor," Appl. Phys. Lett., vol. 73, pp. 780-782, 1998.

[96] C. H. Ahn et al., "Electrostatic modification of novel materials," Rev. Modern Phys., vol. 78, pp. 1185-1212, 2006.

[97] H. Takagi and H. Y. Hwang, "An emergent change of phase for electronics," Science, vol. 327, pp. 1601-1602, 2010.

[98] M. Galiński, A. Lewandowski, and I. Stępniak, "Ionic liquids as electrolytes," 
Electrochimica Acta, vol. 51, pp. 5567-5580, 2006.

[99] T. Fujimoto and K. Awaga, "Electric-double-layer field-effect transistors with ionic liquids," Phys. Chem. Chem. Phys., vol. 15, pp. 8983-9006, 2013.

[100] P. Moetakef et al., "Electrostatic carrier doping of GdTiO3/SrTiO3 interfaces," Appl. Phys. Lett., vol. 99, 2011, Art. ID. 232116.

[101] Y. Lee et al., "Phase diagram of electrostatically doped $\mathrm{SrTiO}_{3}$," Phys. Rev. Lett., vol. 106, 2011, Art. ID. 136809.

[102] Z. Yang, Y. Zhou, and S. Ramanathan, "Studies on room-temperature electric-field effect in ionic-liquid gated $\mathrm{VO}_{2}$ three-terminal devices," J. Appl. Phys., vol. 111, 2012, Art. ID. 014506.

[103] M. Nakano et al., "Collective bulk carrier delocalization driven by electrostatic surface charge accumulation," Nature, vol. 487, pp. 459-462, 2012.

[104] K. Liu et al., "Dense electron system from gate-controlled surface metal-insulator transition," Nano Lett., vol. 12, pp. 6272-6277, 2012.

[105] Y. Zhou and S. Ramanathan, "Relaxation dynamics of ionic liquid- $-\mathrm{VO}_{2}$ interfaces and influence in electric double-layer transistors," J. Appl. Phys., vol. 111, 2012, Art. ID. 084508.

[106] H. Ji, J. Wei, and D. Natelson, "Modulation of the electrical properties of $\mathrm{VO}_{2}$ nanobeams using an ionic liquid as a gating medium," Nano Lett., vol. 12, pp. 2988-2992, 2012.

[107] J. Lu, K. G. West, and S. A. Wolf, "Very large anisotropy in the dc conductivity of epitaxial $\mathrm{VO}_{2}$ thin films grown on (011) rutile $\mathrm{TiO}_{2}$ substrates," Appl. Phys. Lett., vol. 93, 2008, Art. ID. 262107.

[108] A. Gupta et al., "Semiconductor to metal transition characteristics of $\mathrm{VO}_{2}$ thin films grown epitaxially on Si (001)," Appl. Phys. Lett., vol. 95, 2009, Art. ID. 111915.

[109] Y. Zhou and S. Ramanathan, "Heteroepitaxial $\mathrm{VO}_{2}$ thin films on $\mathrm{GaN}$ : Structure and metal-insulator transition characteristics," J. Appl. Phys., vol. 112, 2012, Art. ID. 074114.

[110] D. Ruzmetov, K. T. Zawilski, V. Narayanamurti, and S. Ramanathan, "Structure-functional property relationships in rf-sputtered vanadium dioxide thin films," J. Appl. Phys., vol. 102, 2007, Art. ID. 113715.

[111] D. H. Kim and H. S. Kwok, "Pulsed laser deposition of $\mathrm{VO}_{2}$ thin films," Appl. Phys. Lett., vol. 65, pp. 3188-3190, 1994.

[112] R. Scherwitzl et al., "Electric-field control of the metal-insulator transition in ultrathinNdNiO $\mathrm{O}_{3}$ films," Adv. Mater., vol. 22, pp. 5517-5520, 2010.

[113] C. A. F. Vaz, J. A. Moyer, D. A. Arena, C. H. Ahn, and V. E. Henrich, "Magnetic and electronic structure of ultrathin $\mathrm{La}_{1-\mathrm{x}} \mathrm{Sr}_{\mathrm{x}} \mathrm{MnO}_{3}$ films at half doping," Phys. Rev. B, vol. 90, 2014, Art. ID. 024414.

[114] P. J. Hood and J. F. DeNatale, "Millimeter-wave dielectric properties of epitaxial vanadium dioxide thin films," J. Appl. Phys., vol. 70, pp. 376-381, 1991.

[115] G. Rampelberg et al., "Semiconductor-metal transition in thin $\mathrm{VO}_{2}$ films grown by ozone based atomic layer deposition," Appl. Phys. Lett., vol. 98, 2011, Art. ID. 162902.

[116] T. Blanquart et al., "Atomic layer deposition and characterization of vanadium oxide thin films," RSC Adv., vol. 3, pp. 1179-1185, 2013.

[117] M. B. Sahana, G. N. Subbanna, and S. A. Shivashankar, "Phase transformation and semiconductor-metal transition in thin films of $\mathrm{VO}_{2}$ deposited by low-pressure metalorganic chemical vapor deposition," J. Appl. Phys., vol. 92, pp. 6495-6504, 2002.

[118] T. Maruyama and Y. Ikuta, "Vanadium dioxide thin films prepared by chemical vapour deposition from vanadium(III) acetylacetonate," J. Mater. Sci., vol. 28 , pp. 5073-5078, 1993.

[119] P. Moetakef, J. Y. Zhang, S. Raghavan, A. P. Kajdos, and S. Stemmer, "Growth window and effect of substrate symmetry in hybrid molecular beam epitaxy of a Mott insulating rare earth titanate," $J$. Vacuum Sci. Technol. A, vol. 31, 2013, Art. ID. 041503.

[120] J. W. Tashman et al., "Epitaxial growth of $\mathrm{VO}_{2}$ by periodic annealing," Appl. Phys. Lett., vol. 104, 2014, Art. ID. 063104.

[121] B.-G. Chae et al., "Highly oriented $\mathrm{VO}_{2}$ thin films prepared by sol-gel deposition," Electrochem. Solid-State Lett., vol. 9, pp. C12-C14, 2006.

[122] C. Cen et al., "Nanoscale control of an interfacial metal-insulator transition at room temperature," Nature Mater., vol. 7, pp. 298-302, 2008

[123] T. Kimura et al., "Magnetic control of ferroelectric polarization," Nature, vol. 426, pp. 55-58, 2003.

[124] A. Urushibara et al., "Insulator-metal transition and giant magnetoresistance in $\mathrm{La}_{1-\mathrm{x}} \mathrm{Sr}_{\mathrm{x}} \mathrm{MnO}_{3}$," Phys. Rev. B, vol. 51, pp. 14103-14109, 1995.

[125] H. H. Y. X. Yan-Wu, "Tuning the electrons at the $\mathrm{LaAlO}_{3} / \mathrm{SrTiO}_{3}$ interface: From growth to beyond growth," Chin. Phys. B, vol. 22, 2013, Art. ID. 127301

[126] R. Lopez, T. E. Haynes, L. A. Boatner, L. C. Feldman, and R. F. Haglund, "Size effects in the structural phase transition of $\mathrm{VO}_{2}$ nanoparticles," Phys. Rev. B, vol. 65, 2002, Art. ID. 224113.

[127] J. Wu et al., "Strain-induced self organization of metal-insulator domains in single-crystalline $\mathrm{VO}_{2}$ nanobeams," Nano Lett., vol. 6, pp. 2313-2317, 2006.

[128] S. Zhang, J. Y. Chou, and L. J. Lauhon, "Direct correlation of structural domain formation with the metal insulator transition in a $\mathrm{VO}_{2}$ nanobeam," Nano Lett., vol. 9 , pp. 4527-4532, 2009.

[129] J. I. Sohn et al., "Direct observation of the structural component of the metal-insulator phase transition and growth habits of epitaxially grown $\mathrm{VO}_{2}$ nanowires," Nano Lett., vol. 7, pp. 1570-1574, 2007.

[130] J. M. Baik, M. H. Kim, C. Larson, A. M. Wodtke, and M. Moskovits, "Nanostructure-dependent metal-insulator transitions in vanadium-oxide nanowires," J. Phys. Chem. C, vol. 112, pp. 13 328-13 331, 2008.

[131] R. Jaramillo, F. Schoofs, S. D. Ha, and S. Ramanathan, "High pressure synthesis of $\mathrm{SmNiO}_{3}$ thin films and implications for thermodynamics of the nickelates," J. Mater. Chem. C, vol. 1, pp. 2455-2462, 2013.

[132] J. Shi, Y. Zhou, and S. Ramanathan, "Colossal resistance switching and band gap modulation in a perovskite nickelate by electron doping," Nature Commun. vol. 5, 2014, Art. ID. 5860.
[133] G. W. Burr et al., "Access devices for 3D crosspoint memorya)," J. Vacuum Sci. Technol. B, vol. 32, 2014, Art. ID. 040802.

[134] M. J. Lee et al., "Two series oxide resistors applicable to high speed and high density nonvolatile memory," Adv. Mater., vol. 19, pp. 3919-3923, 2007.

[135] I. P. Radu et al., "Vanadium dioxide for selector applications," ECS Trans., vol. 58, pp. 249-258, 2013

[136] K. Martens et al., "The $\mathrm{VO}_{2}$ interface, the metal-insulator transition tunnel junction, and the metal-insulator transition switch on-off resistance," J. Appl. Phys., vol. 112, 2012, Art. ID. 124501.

[137] X. Liu et al., "Co-occurrence of threshold switching and memory switching in $\mathrm{Pt} / \mathrm{NbO}_{\mathrm{x}} / \mathrm{Pt}$ cells for crosspoint memory applications," IEEE Electron Device Lett., vol. 33, no. 2, pp. 236-238, Feb. 2012.

[138] S. Kim, W. Lee, and H. Hwang, "Selector devices for cross-point ReRAM," in Proc. 13th Int. Workshop Cellular Nanoscale Netw. Their Appl., 2012, DOI: 10.1109/CNNA.2012. 6331466.

[139] E. Cha et al., "Nanoscale $10 \mathrm{~nm}$ 3D vertical ReRAM and $\mathrm{NbO}_{2}$ threshold selector with TiN electrode," in Proc. IEEE Int. Electron Devices Meeting, 2013, pp. 10.5.1-10.5.4.

[140] X. Liu et al., "Reduced threshold current in $\mathrm{NbO}_{2}$ selector by engineering device structure," IEEE Electron Device Lett., vol. 35, no. 10, pp. 1055-1057, Oct. 2014.

[141] X. Liu et al., "Complementary resistive switching in niobium oxide-based resistive memory devices," IEEE Electron Device Lett., vol. 34, no. 2, pp. 235-237, Feb. 2013.

[142] M. Son et al., "Self-selective characteristics of nanoscale devices for high-density ReRAM applications," IEEE Electron Device Lett., vol. 33, no. 5, pp. 718-720, May 2012.

[143] M. Son et al., "Excellent selector characteristics of nanoscale for high-density bipolar ReRAM applications," IEEE Electron Device Lett., vol. 32, no. 11, pp. 1579-1581, Nov. 2011.

[144] S. Kim et al., "Ultrathin $<10 \mathrm{~nm}$ $\mathrm{Nb}_{2} \mathrm{O}_{5} / \mathrm{NbO}_{2}$ hybrid memory with both memory and selector characteristics for high density 3D vertically stackable RRAM applications," in Proc. Symp. VLSI Technol., 2012, pp. 155-156.

[145] H.-S. Wong et al., "Metal-oxide RRAM," Proc. IEEE, vol. 100, no. 6, pp. 1951-1970, Jun. 2012.

[146] K. M. Kim, D. S. Jeong, and C. S. Hwang, "Nanofilamentary resistive switching in binary oxide system; A review on the present status and outlook," Nanotechnology, vol. 22, 2011, Art. ID. 254002.

[147] I. G. Baek et al., "Highly scalable nonvolatile resistive memory using simple binary oxide driven by asymmetric unipolar voltage pulses," in IEDM Tech. Dig. IEEE Int. Electron Devices Meeting, 2004, pp. 587-590.

[148] K. Tsunoda et al., "Low power and high speed switching of Ti-doped NiO ReRAM under the unipolar voltage source of less than $3 \mathrm{~V}$," in Proc. IEEE Int. Electron Devices Meeting, 2007, pp. 767-770.

[149] L. F. Liu et al., "Current compliance-free resistive switching in nonstoichiometric $\mathrm{CeO}_{\mathrm{x}}$ films for nonvolatile memory application," in Proc. IEEE Int. Memory Workshop, 2009, DOI: 10.1109/IMW. 2009.5090586.

[150] H. B. Lv et al., "Resistive memory switching of $\mathrm{Cu}_{\mathrm{x}} \mathrm{O}$ films for a nonvolatile 
memory application," IEEE Electron Device Lett., vol. 29, no. 4, pp. 309-311, Apr. 2008.

[151] K. Sungho, M. Hanul, D. Gupta Y. Seunghyup, and Y.-K. Choi, "Resistive switching characteristics of sol-gel zinc oxide films for flexible memory applications," IEEE Trans. Electron Devices, vol. 56, no. 4, pp. 696-699, Apr. 2009.

[152] H. Y. Lee et al., "Low power and high speed bipolar switching with a thin reactive $\mathrm{Ti}$ buffer layer in robust $\mathrm{HfO}_{2}$ based RRAM," in Proc. IEEE Int. Electron Devices Meeting, 2008, DOI: 10.1109/IEDM.2008.4796677.

[153] S. Seo et al., "Reproducible resistance switching in polycrystalline $\mathrm{NiO}$ films," Appl. Phys. Lett., vol. 85, pp. 5655-5657, 2004.

[154] T. Sakata, K. Sakata, G. Höfer, and T. Horiuchi, "Preparation of $\mathrm{NbO}_{2}$ single crystals by chemical transport reaction," J. Crystal Growth, vol. 12, pp. 88-92, 1972 .

[155] S. D. Ha, Z. You, A. E. Duwel, D. W. White, and S. Ramanathan, "Quick switch: Strongly correlated electronic phase transition systems for cutting-edge microwave devices," IEEE Microw. Mag., vol. 15, no. 6, pp. 32-44, Sep./Oct. 2014.

[156] M. D. Pickett, J. Borghetti, J. J. Yang, G. Medeiros-Ribeiro, and R. S. Williams, "Coexistence of memristance and negative differential resistance in a nanoscale metal-oxide-metal system," Adv. Mater. vol. 23, pp. 1730-1733, 2011.

[157] M. Son et al., "Self-selective characteristics of nanoscale $\mathrm{VO}_{\mathrm{x}}$ devices for high-density ReRAM applications," IEEE Electron Device Lett., vol. 33, no. 5, pp. 718-720, May 2012.

[158] A. S. Barker, H. W. Verleur, and H. J. Guggenheim, "Infrared optical properties of vanadium dioxide above and below the transition temperature," Phys. Rev. Lett., vol. 17, pp. 1286-1289, 1966.

[159] A. Zylbersztejn, B. Pannetier, and P. Merenda, "Fast pulse measurements of the dielectric constant of semiconducting $\mathrm{VO}_{2}$," Phys. Lett. A, vol. 54, pp. 145-147, 1975.

[160] Z. Yang, C. Ko, V. Balakrishnan, G. Gopalakrishnan, and S. Ramanathan, "Dielectric and carrier transport properties of vanadium dioxide thin films across the phase transition utilizing gated capacitor devices," Phys. Rev. B, vol. 82, 2010 Art. ID. 205101.

[161] M. Kim et al., "A new single element phase transition memory," in Proc. 10th IEEE Conf. Nanotechnol., 2010, pp. 439-442.

[162] S. H. Lee et al., "Vanadium dioxide $\left(\mathrm{VO}_{2}\right)$ is also a ferroelectric: Properties from memory structures," in Proc. 11th IEEE Conf. Nanotechnol., 2011, pp. 735-739.

[163] H. Sang et al., "Space charge polarization induced memory in $\mathrm{SmNiO}_{3} / \mathrm{Si}$ transistors," Appl. Phys. Lett., vol. 102 2013, Art. ID. 072102.

[164] T. Driscoll, H.-T. Kim, B.-G. Chae, M. Di Ventra, and D. N. Basov, "Phase-transition driven memristive system," Appl. Phys. Lett., vol. 95, 2009, Art. ID. 043503.

[165] T. Driscoll et al., "Memory metamaterials," Science, vol. 325, pp. 1518-1521, 2009.

[166] Y.-G. Jeong et al., "Electrical control of terahertz nano antennas on $\mathrm{VO}_{2}$ thin film," Opt. Exp., vol. 19, pp. 21 211-21 215, 2011.
[167] D. J. Shelton, K. R. Coffey, and G. D. Boreman, "Experimental demonstration of tunable phase in a thermochromic infrared-reflectarray metamaterial," Opt. Exp., vol. 18, pp. 1330-1335, 2010.

[168] M. J. Dicken et al., "Frequency tunable near-infrared metamaterials based on $\mathrm{VO}_{2}$ phase transition," Opt. Exp., vol. 17, pp. 18 330-18 339, 2009.

[169] A. K. Jain, J. Mao, and K. Mohiuddin, "Artificial neural networks: A tutorial," Computer, vol. 29, pp. 31-44, 1996.

[170] IBM Blue Gene Team, "Overview of the IBM Blue Gene/P project,” IBM J. Res. Develop., vol. 52, pp. 199-220, 2008.

[171] Q. V. Le, "Building high-level features using large scale unsupervised learning," in Proc. IEEE Int. Conf. Acoust. Speech Signal Process., 2013, pp. 8595-8598.

[172] R. Ananthanarayanan, S. K. Esser, H. D. Simon, and D. S. Modha, "The cat is out of the bag: Cortical simulations with 109 neurons, 1013 synapses," in Proc. Conf. High Performance Comput. Netw. Storage Anal., 2009, DOI: 10.1145/ 1654059.1654124.

[173] K. Minkovich, N. Srinivasa, J. M. Cruz-Albrecht, C. Youngkwan, and A. Nogin, "Programming time-multiplexed reconfigurable hardware using a scalable neuromorphic compiler," IEEE Trans. Neural Netw. Learn. Syst., vol. 23, no. 6, pp. 889-901, Jun. 2012.

[174] P. A. Merolla et al., "A million spiking-neuron integrated circuit with a scalable communication network and interface," Science, vol. 345, pp. 668-673, 2014.

[175] D. Kuzum, S. Yu, and H. S. P. Wong, "Synaptic electronics: Materials, devices and applications," Nanotechnology, vol. 24, 2013, Art. ID. 382001.

[176] T. Sharp, F. Galluppi, A. Rast, and S. Furber, "Power-efficient simulation of detailed cortical microcircuits on SpiNNaker," J. Neurosci. Methods, vol. 210, pp. 110-118, 2012.

[177] K.-H. Kim et al., "A functional hybrid memristor crossbar-array/CMOS system for data storage and neuromorphic applications," Nano Lett., vol. 12, pp. 389-395, 2012.

[178] A. L. Hodgkin and A. F. Huxley, "A quantitative description of membrane current and its application to conduction and excitation in nerve," J. Physiol. vol. 117, pp. 500-544, 1952.

[179] M. D. Pickett, G. Medeiros-Ribeiro, and R. S. Williams, "A scalable neuristor built with Mott memristors," Nature Mater. vol. 12, pp. 114-117, 2013.

[180] J. Nagumo, S. Arimoto, and S. Yoshizawa, "An active pulse transmission line simulating nerve axon," Proc. IRE, vol. 50, pp. 2061-2070, 1962.

[181] J.-I. Nishizawa and A. Hayasaka, "Two-line neuristor with active element in series and in parallel†," Int. J. Electron., vol. 26, pp. 437-469, 1969.

[182] H. Markram, J. Lübke, M. Frotscher, and B. Sakmann, "Regulation of synaptic efficacy by coincidence of postsynaptic APs and EPSPs," Science, vol. 275, pp. 213-215, 1997.

[183] G.-Q. Bi and M.-M. Poo, "Synaptic modifications in cultured hippocampal neurons: Dependence on spike timing, synaptic strength, and postsynaptic cell type," J. Neurosci., vol. 18, pp. $10464-10472,1998$.

[184] F. Alibart, E. Zamanidoost, and D. B. Strukov, "Pattern classification by memristive crossbar circuits using ex situ and in situ training," Nature Commun., vol. 4, 2013, Art. ID. 2032.

[185] C. Diorio, P. Hasler, B. A. Minch, and C. A. Mead, "A single-transistor silicon synapse," IEEE Trans. Electron Devices, vol. 43, no. 11, pp. 1972-1980, Nov. 1996.

[186] J. Shi, S. D. Ha, Y. Zhou, F. Schoofs, and S. Ramanathan, "A correlated nickelate synaptic transistor," Nature Commun., vol. 4, 2013, Art. ID. 3676

[187] S. D. Ha, J. Shi, Y. Meroz, L. Mahadevan, and S. Ramanathan, "Neuromimetic circuits with synaptic devices based on strongly correlated electron systems," Phys. Rev. Appl., vol. 2, 2014, Art. ID. 064003.

[188] D. Loke et al., "Breaking the speed limits of phase-change memory," Science, vol. 336, pp. 1566-1569, 2012.

[189] M. Rini et al., "Control of the electronic phase of a manganite by mode-selective vibrational excitation," Nature, vol. 449, pp. 72-74, 2007.

[190] M. K. Liu et al., "Photoinduced phase transitions by time-resolved far-infrared spectroscopy in $\mathrm{VO}_{2}$," Phys. Rev. Lett., vol. 107, 2011, Art. ID. 066403.

[191] M. F. Becker et al., "Femtosecond laser excitation of the semiconductor-metal phase transition inVO 2 ," Appl. Phys. Lett., vol. 65, pp. 1507-1509, 1994.

[192] L. A. Gea and L. A. Boatner, "Optical switching of coherent $\mathrm{VO}_{2}$ precipitates formed in sapphire by ion implantation and annealing," Appl. Phys. Lett., vol. 68 , pp. 3081-3083, 1996

[193] C. Kübler et al., "Coherent structural dynamics and electronic correlations during an ultrafast insulator-to-metal phase transition in $\mathrm{VO}_{2}$," Phys. Rev. Lett., vol. 99, 2007, Art. ID. 116401.

[194] M. Nakajima, N. Takubo, Z. Hiroi, Y. Ueda, and T. Suemoto, "Photoinduced metallic state in $\mathrm{VO}_{2}$ proved by the terahertz pump-probe spectroscopy," Appl. Phys. Lett., vol. 92, 2008, Art. ID. 011907.

[195] H.-T. Kim et al., "Monoclinic and correlated metal phase in $\mathrm{VO}_{2}$ as evidence of the Mott transition: Coherent phonon analysis," Phys. Rev. Lett., vol. 97, 2006, Art. ID. 266401.

[196] M. Liu et al., "Terahertz-field-induced insulator-to-metal transition in vanadium dioxide metamaterial," Nature, vol. 487. pp. 345-348, 2012.

[197] K. Appavoo et al., "Ultrafast phase transition via catastrophic phonon collapse driven by plasmonic hot-electron injection," Nano Lett., vol. 14, pp. 1127-1133, 2014.

[198] S. Lysenko et al., "Light-induced ultrafast phase transitions in $\mathrm{VO}_{2}$ thin film," Appl. Surface Sci., vol. 252, pp. 5512-5515, 2006.

[199] S. Lysenko, A. Rúa, V. Vikhnin, F. Fernández, and H. Liu, "Insulator-to-metal phase transition and recovery processes in $\mathrm{VO}_{2}$ thin films after femtosecond laser excitation," Phys. Rev. B, vol. 76, 2007, Art. ID. 035104.

[200] M. Liu et al., "Terahertz-field-induced insulator-to-metal transition in vanadium dioxide metamaterial," Nature, vol. 487, pp. 345-348, 2012.

[201] L. Perfetti et al., "Time evolution of the electronic structure of $1 \mathrm{~T}-\mathrm{TaS}_{2}$ through the insulator-metal transition," Phys. Rev. Lett., vol. 97, 2006, Art. ID. 067402.

[202] B.-G. Chae, H.-T. Kim, D.-H. Youn, and K.-Y. Kang, "Abrupt metal-insulator 
transition observed in $\mathrm{VO}_{2}$ thin films induced by a switching voltage pulse," Physica $B$, Condensed Matter, vol. 369, pp. 76-80, 2005

[203] R. Servin et al., "Unravelling the switching mechanisms in electric field induced insulator-metal transitions in $\mathrm{VO}_{2}$ nanobeams," J. Phys. D, Appl. Phys. vol. 47, 2014, Art. ID. 295101.

[204] J. S. Brockman et al., "Subnanosecond incubation times for electric-field-induced metallization of a correlated electron oxide," Nature Nanotechnol., vol. 9, pp. 453-458, 2014.

[205] G. Seo et al., "Voltage-pulse-induced switching dynamics in $\mathrm{VO}_{2}$ thin-film devices on silicon," IEEE Electron Device Lett., vol. 32, no. 11, pp. 1582-1584, Nov. 2011.

[206] A. Kar et al., "Intrinsic electronic switching time in ultrathin epitaxial vanadium dioxide thin film," Appl. Phys. Lett., vol. 102, 2013, Art. ID. 072106.

[207] Y. Zhang and S. Ramanathan, "Analysis of "on" and "off" times for thermally driven $\mathrm{VO}_{2}$ metal-insulator transition nanoscale switching devices," Solid-State Electron., vol. 62, pp. 161-164, 2011.

[208] S. Hormoz and S. Ramanathan, "Limits on vanadium oxide Mott metal-insulator transition field-effect transistors,"

Solid-State Electron., vol. 54, pp. 654-659, 2010.
[209] K. T. Jacob, C. Shekhar, M. Vinay, and Y. Waseda, "Thermodynamic properties of niobium oxides," J. Chem. Eng. Data, vol. 55, pp. 4854-4863, 2010.

[210] C. N. Berglund and H. J. Guggenheim, "Electronic properties of $\mathrm{VO}_{2}$ near the semiconductor-metal transition," Phys. Rev., vol. 185, pp. 1022-1033, 1969.

[211] D.-W. Oh, C. Ko, S. Ramanathan, and D. G. Cahill, "Thermal conductivity and dynamic heat capacity across the metal-insulator transition in thin film $\mathrm{VO}_{2}$," Appl. Phys. Lett., vol. 96, 2010, Art. ID. 151906.

\section{ABOUT THE AUTHORS}

You Zhou received the B.S. degree in physics from Peking University, Beijing, China, in 2010 and the Ph.D. degree in applied physics from Harvard University, Cambridge, MA, USA, in 2015.

He is currently a Postdoctoral Fellow in the John A. Paulson School of Engineering and Applied Sciences, Harvard University. His research has focused on the material physics of complex oxides and their applications to electronic and optical devices as well as energy conversion.

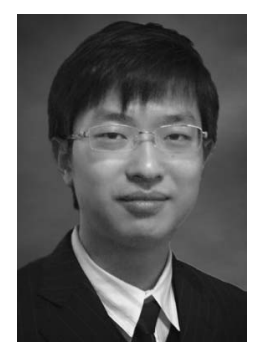

Shriram Ramanathan received the Ph.D. degree in materials science and engineering from Stanford University, Stanford, CA, USA, in 2002. His doctoral dissertation was carried out under the supervision of P. C. Mclntyre.

He subsequently joined the research staff at Components Research, Intel, and worked on realization of 3-D integrated circuits and highresolution noninvasive microscope design for over three years. He has since served on the

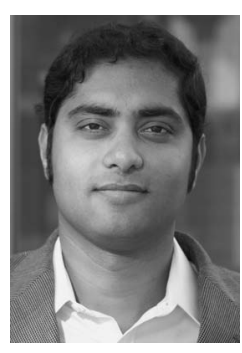
Applied Physics faculty at Harvard University, Cambridge, MA, USA. His research interests are in the areas of thin-film oxide materials science and solid-state devices. 